\title{
Applying System Dynamics to Simulate the Passenger Flow in Subway Stations
}

\author{
Jianghua Gao, ${ }^{1,2}$ Limin Jia $\mathbb{D}^{2,3}$ and Jianyuan Guo $\mathbb{D}^{1,3}$ \\ ${ }^{1}$ School of Traffic and Transportation, Beijing Jiaotong University, Beijing 100044, China \\ ${ }^{2}$ State Key Lab of Rail Traffic Control \& Safety, Beijing Jiaotong University, Beijing 100044, China \\ ${ }^{3}$ Beijing Research Center of Urban Traffic Information Sensing and Service Technologies, Beijing Jiaotong University, \\ Beijing 100044, China \\ Correspondence should be addressed to Jianyuan Guo; jyguo@bjtu.edu.cn
}

Received 9 August 2019; Accepted 14 September 2019; Published 19 December 2019

Academic Editor: Maria Alessandra Ragusa

Copyright (c) 2019 Jianghua Gao et al. This is an open access article distributed under the Creative Commons Attribution License, which permits unrestricted use, distribution, and reproduction in any medium, provided the original work is properly cited.

\begin{abstract}
A macroscopic passenger flow simulation model based on system dynamics is proposed in this paper. It considers the key factors influencing the dynamics of passenger flow from a holistic perspective of the stations and then models the dynamic change in the number of passengers. Firstly, the transmission of passenger flow for a general many-to-many relation between nodes are presented. When the sum of sending capacities heading for a downstream node is less than the receiving capacity of this node, the aggregation of stranded passengers will form the queuing part of the node. The results coming from a passenger flow simulation of a subway station in Beijing show that the proposed model performs well by comparing it with the real data. It can be applied to describe the dynamic change in the number of passengers and the level of service for the facilities.
\end{abstract}

\section{Introduction}

The ever-increasing growth in urban rail transit demand implies the increasing demand for its service, which requires more efficient services in stations. As the critical place for passengers entering and leaving the urban rail transit system, the station provides passengers with a lot of services such as security checks and transferring from one platform to another. It often suffers from relatively heavy congestion with a large scale of passengers gathering on a series of facilities, which results in discomfort to passengers and more likely gives rise to some unsafety. Obtaining the number of passengers and their changes in various facilities is the basic but most important way to keeping a good level of service (LOS) [1] and ensuring the safeties of passengers [2].

A central challenge in passenger flow modeling consists in utilizing appropriate indicators to evaluate the level of passenger flow. The LOS is applied to study the degree of congestion on LRT (Light Rail Transit) platforms, and the degree of crowding in a vehicle. With the above two congestion or crowding indicators, the relationships between the dwelling time of trains and the crowding situations at LRT stations are firstly determined [1]. The total evacuation time and maximum flow capacity of the selected staircase that directly connects with the platform is utilized to evaluate the evacuation process of the alighting passengers in subway stations during rush hours [3]. The entropy of velocity, which represents both the motion magnitude distribution and the motion direction distribution, is firstly introduced to detect the congestion [4].

Many researches concentrate on the field of subway station generally considering the complexity of station layout, variety of facilities, heterogeneity, and behavioral diversity of passengers and interactions between passengers and station environment [5]. Simulation method has been introduced to the study of passenger flow in stations, which is helpful not only to obtain the LOS and safety in stations but also to conduct station management including passenger flow evacuation and control. Simulation models can be broadly classified into microscopic models and macroscopic models. These microscopic models are the spatial-discrete models such as the cellular automation model [6] and the spatial-continuous models such as the social force model $[7,8]$. Macroscopic models cover the continuum model [9], the cell transmission model 
$[10,11]$, the link transmission model [12], the queuing model [13], the system dynamics model [14], etc.

Microscopic models mainly emphasize on the description of individual behavior to reproduce some self-organized phenomena [7]. It is widely applied to the simulation of facilities with specific physical attributes or to that of several flow patterns such as the bottleneck flow and the counter-flow. The application of microscopic methods in a subway station is generally to simulate passenger flow through some specific facilities, such as staircases, escalators, or a group of facilities with a particular function such as the exchange of passengers during alighting and boarding trains. Ref. [15] applies a modified social force model to describe the three-dimensional movement of individuals on the staircase in the subway station, taking into consideration the calibration of parameters such as walking speed, body size, etc. Ref. [16] puts forward a new application of cellular automata model to simulate the alighting and boarding movement of individuals in subway stations, in which the transition probabilities for modeling passenger cooperation and negotiation can be obtained by combining these important factors including individual desire, pressure from passengers behind, personal activity and tendencies. Both $[17,18]$ aim at obtaining bidirectional pedestrian flow fundamental diagram using cellular automata. Besides this, many simulation tools are developed based on these microscopic methods. For example, pedestrian library in AnyLogic based on social force model $[5,19]$ generally simulates the passenger flow in normal circulation; building exodus applied in [20] mainly concentrates on evacuation scenario. However, there exist some drawbacks in practical use: one is taking too much time to design simulating experiment for a complicated environment with a big surface, variety of facilities, etc.; another is being short of flexibility for different environmental scenarios. Besides, they are computationally costly in dealing with a large-scale of passengers.

Macroscopic methods operate at the level of aggregation and tend to omit plenty of details on individual behaviors. In the cell transmission model, each of the facilities in the studied space will be discretized into a set of uniform cells of the same size, which could give a rather rapid construction of a simulating environment with complex geometry. Building on the continuum theory and the cell transmission model, Ref. [20] put forward a dynamic network-loading model at the macroscopic level, with typical applications to several distinct flow patterns such as counter-flow, cross-flow, and bottleneck flow, and another two practical applications to a Swiss railway station and a Dutch bottleneck flow. The link transmission model $[21,22]$ has an extensive application in road traffic for the regular geometry of the road links. However, to the best of our knowledge, it is inappropriate for the studies in subway stations with multi-directional flow, and various or irregular geometry. Most queuing models [23] mainly focus on modeling of individual facilities in stations, by regarding facilities and passengers as service desks and customers, respectively, and it perform well in describing the queuing process. Ref. [24] presents the $\mathrm{PH} / \mathrm{PH}(\mathrm{n}) / \mathrm{C} / \mathrm{C}$ model based on phase-type distribution which can approach any positive variable to study the arrivals of passengers. For system dynamics, the modeling of the passenger flow [25] encompasses two phases. Firstly, from a holistic perspective, it incorporates all of the key factors such as topology of the simulation environment, and characteristic of passenger flow, which are presented in the causal-loop diagram to show how elements in every factor interact with each other. Secondly, the stock-flow diagram is used to simulate the time-varying change of these elements in indexes, such as volume, density, and queuing length.

Macroscopic passenger flow models can be broadly distinguished into network-based and network-free models. One of the most widely used network-based models is the queueing network, which considers passengers at the disaggregate level and presenting space based on graph theory. Ref. [26] builds the queuing network based on modeling of each facility by considering the relationship between them. On the contrary, most network-free models Ref. [27] are based on continuum theory for passenger flow, which is formulated as several partial differential equations. The magnitude of passenger flow velocity is determined by the speed obtained from the passenger flow fundamental diagram relating speed and density. The direction of passenger flow depends on the dynamics of potential fields, defined for a specific group of passengers in the same area with the same destination. For, the computation cost seems to increase enormously for these potential fields [28], which, as far as [20] are concerned, is why this approach has attracted little interest in the large-scale applications with complicated networks.

In this paper, we select an appropriate model to simulate the passenger flow in stations based on the following reasons. First, the information of the layout, variety of facilities, and demand with a specific destination in subway stations being operated are relatively exact and can be known a priori. Besides, the macroscopic models are much more appropriate for the modeling of passenger behaviors at the aggregate level, compared with the microscopic models, which mainly focus on the detailed movements of individuals [29].

So the network-based macroscopic model incorporating system dynamics [30] proposed in this paper with the following three reasons. Firstly, the simulation of a subway station with a complicated layout and high-density passenger flows during a long period can be accomplished efficiently, with most models dealing with a low-density passenger flow [31]. Secondly, allowing for the coordination between sending capacities of upstream nodes and receiving capacities of downstream nodes [11] can give a reasonable explanation for the mechanism of the passenger flow transmission between nodes. Thirdly, from the level of aggregation, the movements depending on the fundamental diagram relating to speed and density [32] are suitable for high-density passenger flows. Finally, after comparing the queuing space with the node space in size, physical queue [33] is applied to describe the space occupied by the passengers being stranded due to the constraint of capacity [34] of the downstream nodes. This methodology can be extended to the applications in biological field $[35,36]$.

The structure of this paper is as follows. Section 2 presents the problem description. Section 3 describes the simulation model based on system dynamics. Section 4 shows the case study of a real station to verify the effectiveness and applicability of the proposed model. Finally, conclusions and directions for future research are made. 


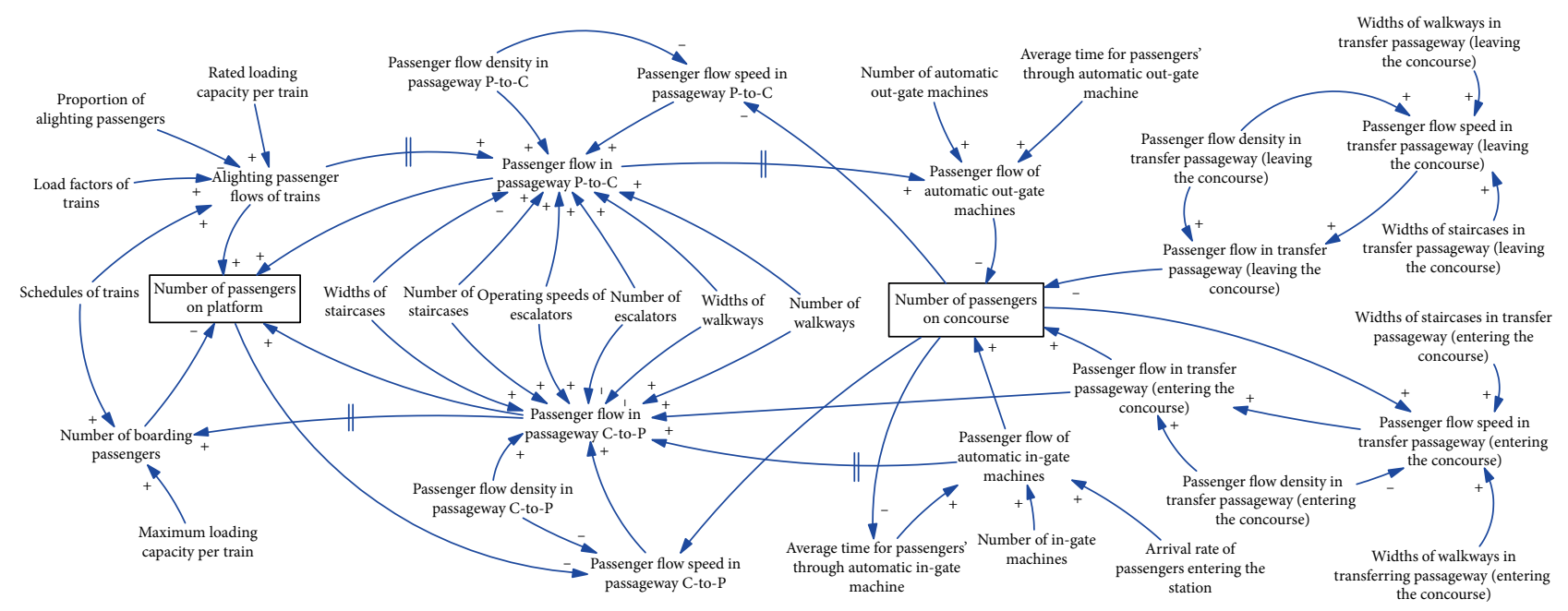

FIGURE 1: Causal-loop diagram for the transfer station. Facilities in the unpaid zone are excluded from the range of study. P-to-C represents the flow direction from the platform to the concourse and C-to-P vice versa. The walkway is different from the passageway; the former is one kind of facilities, and the latter represents a set of serial facilities including escalators, staircases, and walkways.

\section{Problem Description}

In the section, after analyzing a variety of facilities, gathering and scattering process, we present the key factors on the performance of the subway station and then established the casual-loop diagram.

2.1. A Variety of Facilities. Facilities have direct influences on the movements of the passengers walking along it and on the distribution of passengers in the whole subway station. There exist a variety of facilities in the subway station, which can be classified into holding, flow, and processing facilities. Holding facility referring to the platform, concourse, and waiting areas of other facilities provides passengers with a certain space. It mainly presents the static behaviors of passengers but also involves some dynamic characteristics of passenger flow, considering the circulation on it and its connection with other types of facilities. Flow facilities such as staircases and walkways mainly depend on the length, width, and slope etc. The characteristics of passengers along the facility affect it significantly. Processing facilities provide certain services (e.g., automatic ticket check, security inspection, passenger transport, ticket purchases, etc.) to passengers using specific facilities (e.g., automatic ticket checker also called as gate machine, automatic security inspection machine, escalator, ticket vending machine, etc.).

2.2. Gathering and Scattering Process. The gathering and scattering process involves arrival-boarding and alightingdeparture process, and it includes a transferring-boarding process for the transfer station. The arrival-boarding process starts typically from in-gates and finishes when passengers leave the platform and board on to the train. The alightingdeparture process is similar to the arrival-boarding process but in a different direction. It starts from the train and finishes when passengers go outside of the station. The transferring-boarding process starts from the train operating on one subway line and finishes when passengers board another train running on another subway line. These three processes are for passengers with different destinations in the station. Some facilities may be involved in more than one process at the same time, such as the staircase and the concourse, which improves the efficiency of them but results in the complicated layout of facilities.

2.3. Causal-Loop Diagram for the Transfer Station. As one of the complex feedback systems, the subway station is influenced by five key factors covering train operation organization, the layout of the station, the station management, the organization, the passengers, and the facilities [30]. As shown in Figure 1, taking the transfer station as an example, a causal-loop diagram is formed by integrating these five key factors with corresponding elements.

Train operation organization based on the schedules of trains, generally involving elements such as load factors, intervals, and dwelling times of trains, determines how many passengers and how frequently they will be transported from the current station to others and vice versa. For station management, measures in passenger flow control such as the limitation of passenger flow and adjustments of paths are taken to optimize the distribution of passengers in the station, allowing for the existing station layout and passenger demands. A variety of passengers with different destinations in the station affect the mobility of passenger flow. As described in Section 2.1 , facilities with different physical parameters such as width and length are the critical influencing factors on the number of passengers being served by them and the duration time of passengers along them. One of the typical feedback relationships is presented by the relations between flow, density, and speed in the fundamental diagram of passenger flow.

\section{A Simulation Model Based on System Dynamics}

We consider discrete-time, i.e., each instant of the entire simulation time $T$ is presented as $\tau=\{0,1, \cdots, T / \Delta \tau\}$ with 


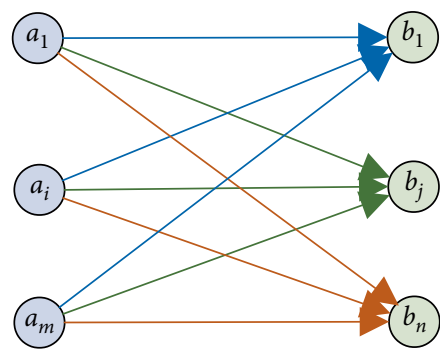

FIGURE 2: Schematic to a general connection between upstream and downstream nodes.

uniform time interval $\Delta \tau$. The station network is composed of nodes, where a node can represent either a facility such as a staircase and an escalator serving for unidirectional or bidirectional passenger flow, or a part of a facility such as a platform and a concourse for multi-directional passenger flow.

System dynamics is applied to present the time-varying change of passenger flow for the whole station based on the causal-loop diagram in Figure 1. The number of passengers in a node during time interval $\Delta \tau$ at time $\tau+1$ can be expressed as

$$
n(\tau+1)=n(\tau)+\sum_{i}\left(f_{i}^{\text {in }}(\tau)-f_{j}^{\text {out }}(\tau)\right),
$$

where $f_{i}^{\text {in }}(\tau)$ and $f_{j}^{\text {out }}(\tau)$ are the actual $i$ th inflow and $j$ th outflow of the current node during time interval $\Delta \tau$ at time $\tau$, respectively.

In the subway station, there exist several kinds of relations between nodes, including one-to-one, one-to-many, many-toone, and many-to-many; however, the first three can be considered as the specific forms of the fourth. To acquire the number of passengers in Equation (1), the actual inflow and outflow of the current node can be determined by the following passenger flow transmission relationship. Figure 2 shows the general connection between nodes with splitting and merging of passenger flows, where an upstream node $a_{i}$ has a downstream node $b_{j} \in B_{a_{i}}$, and a downstream node $b_{j}$ has an upstream node $a_{i} \in A_{b}$.

A sending and receiving capacity, representing the maximum number of passengers who can leave and enter a node, is specified for each node, referring to [11] that the original cell transmission model is proposed.

The sending capacity of the node $a_{i}$ to nodes $b_{j} \in B_{a_{i}}$ during time interval $\Delta \tau$ at time $\tau$ is given by

$$
S_{a_{i} \rightarrow b_{j}}(\tau)=\delta_{a_{i} \rightarrow b_{j}}(\tau) \min \left\{x_{a_{i}}^{\text {out }}(\tau), C_{a_{i}}^{\text {out }}(\tau)\right\},
$$

where $\delta_{a_{i} \rightarrow b_{j}}(\tau)$ is the splitting proportion corresponding to the link $a_{i} \rightarrow b_{j}$ for the ensemble of passengers in node $a_{i}$ during time interval $\Delta \tau$ at time $\tau$. The hydrodynamic outflow capacity $C_{a_{i}}^{\text {out }}(\tau)$ can be obtained by passenger flow fundamental diagram and the approach to dealing with the passenger flow in cells referring to [37] will be applied in the paper. It must be noted that different kinds of facilities have different passenger flow fundamental diagrams. $x_{a_{i}}^{\text {out }}(\tau)$ is the number of passengers waiting to leave node $a_{i}$ during time interval $\Delta \tau$, and can be obtained by Equation (13).

For node $b_{j}$ during time interval $\Delta \tau$ at time $\tau$, the receiving capacity can be expressed by

$$
R_{b_{j}}^{i n}(\tau)=\min \left\{n_{b_{j}}^{\max }-n_{b_{j}}(\tau), C_{b_{j}}^{\text {in }}(\tau)\right\},
$$

where $n_{b_{i}}^{\max }$ is the holding capacity that is assumed to be the maximum number of passengers that can be accommodated by node $b_{j}$ at jam density. $n_{b}(\tau)$ is the actual number of passengers in the node $b_{j}$ during time interval $\Delta \tau$ at time $\tau$. $C_{b_{j}}^{\text {in }}(\tau)$ is the hydrodynamic inflow capacity denoting the maximum number of passengers that can enter node $b_{j}$ during time interval $\Delta \tau$ according to the fundamental diagram [37].

The actual flow of passengers from the upstream node $a_{i}$ to the downstream node $b_{j}$ during time interval $\Delta \tau$ at time $\tau$ can be expressed as

$$
F_{a_{i} \rightarrow b_{j}}(\tau)= \begin{cases}S_{a_{i} \rightarrow b_{j}}(\tau), & \text { if } \sum_{a_{i}} S_{a_{i} \rightarrow b_{j}}(\tau) \leq R_{b_{j}}^{i n}(\tau), \\ \xi_{a_{i} \rightarrow b_{j}}(\tau) \cdot R_{b_{j}}^{\text {in }}(\tau), & \text { otherwise. }\end{cases}
$$

If the receiving capacity for node $b_{j}$ is superior to the sum of sending capacities heading for it, the actual flow equals the sending capacity. Otherwise, the various inflowing passengers are determined by the allocation of the receiving capacity in proportion [37]. The allocation of the receiving capacity is then given by

$$
\xi_{a_{i} \rightarrow b_{j}}(\tau)=\frac{S_{a_{i} \rightarrow b_{j}}(\tau)}{\sum_{a_{i}} S_{a_{i} \rightarrow b_{j}}(\tau)} .
$$

The actual outflow of node $a_{i}$ during time interval $\Delta \tau$ at time $\tau$ can be determined as

$$
f_{a_{i}}^{\text {out }}(\tau)=\sum_{b_{j}} F_{a_{i} \rightarrow b_{j}}(\tau)
$$

while the actual inflow of node $b_{j}$ during time interval $\Delta \tau$ at time $\tau$ can be determined as

$$
f_{b_{j}}^{i n}(\tau)=\sum_{a_{i}} F_{a_{i} \rightarrow b_{j}}(\tau) .
$$

In regard to the capacity constraints of the downstream nodes, the aggregation of passenger flow will form at the exit of the current node, as can be known from Equation (5). For formulation convenience, the whole node can be divided into two adjacent time-varying parts: one is walking area, and the other is the queuing area. It should be noted that the aggregation of passengers will be generally referred to as queuing that will be classified into disordered queuing and ordered queuing, as shown in Figures 3 and 4 respectively. Combining with Equation (6), after one transmission for node $a_{i}$ during time interval $\Delta \tau$ at time $\tau+1$, the number of passengers being stranded can be expressed by

$$
y_{a_{i}}^{\text {out }}(\tau+1)=y_{a_{i}}^{\text {out }}(\tau)-f_{a_{i}}^{\text {out }}(\tau)
$$

In comparison with the space of facilities, the space occupied by queuing passengers cannot be ignored, that is why physical queue [38], instead of point queue [39], will be used in the following.

For disordered queuing, the queuing length at node $a_{i}$ formed by stranded passengers at time $\tau+1$ is given by

$$
l_{a_{i}, q}(\tau+1)=\frac{y_{a_{i}}^{\text {out }}(\tau+1)}{w_{a_{i}} k_{a_{i} j a m}}
$$




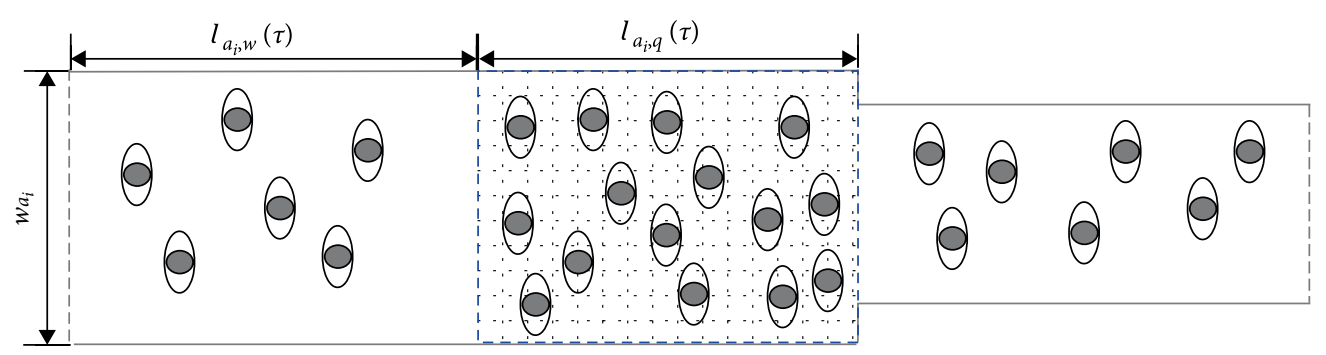

FIGURE 3: Disordered queuing.

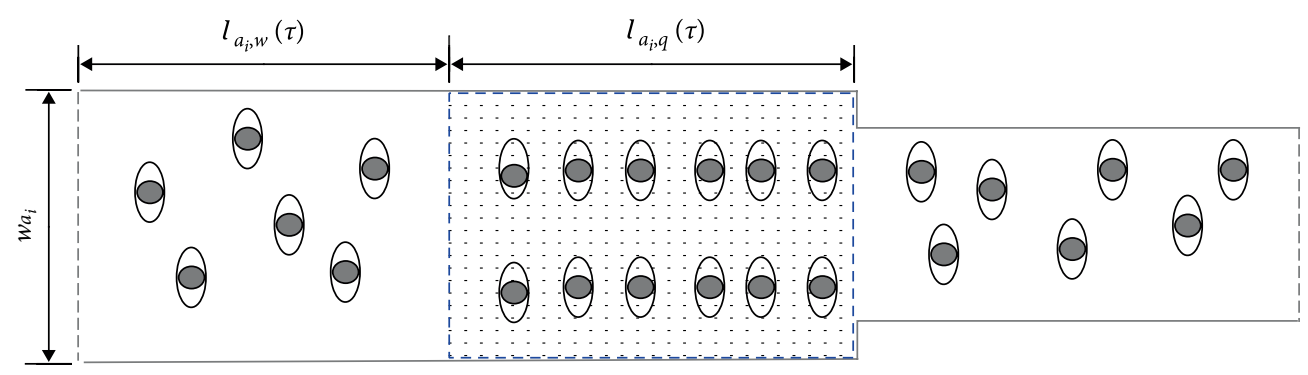

FIGURE 4: Ordered queuing.

where $k_{a_{i}, \text { jam }}$ is the jam density at which the magnitude of passenger flow speed reaches $0 . w_{a_{i}}$ is the physical width of the node $a_{i}$.

For ordered queuing, the queuing length at node $a_{i}$ formed by stranded passengers at time $\tau+1$ is given by

$$
l_{a_{i}, q}(\tau+1)=\frac{y_{a_{i}}^{\text {out }}(\tau+1)}{\Delta l_{a_{i}}} \xi
$$

$\Delta l_{a_{i}}$ is the average distance between two adjacent passengers in node $a_{i}$. $\xi$ is the number of queues under ordered queuing.

For a group of passengers, its entering time into the walking area must be before $\tau+1-\left\lceil t_{a_{i}}(\tau+1) / \Delta \tau\right\rceil$, if it leaves the walking area into the queuing area of node $a_{i}$ at the end of time $\tau+1 . t_{a_{i}}(\tau+1)$ is the walking time in the walking area for this group of passengers, i.e., the time intervals needed are $\left.t_{a_{i}}(\tau+1) / \Delta \tau\right\rceil$ denoted as $t_{a_{i}}^{\Delta \tau}$. The cumulative passenger flow has the following relationship:

$$
f_{a_{i}, w}^{o u t}(\tau)=f_{a_{i}}^{\text {in }}\left(\tau+1-t_{a_{i}}^{\Delta \tau}\right)
$$

where $f_{a_{i}}^{\text {in }}\left(\tau+1-t_{a_{i}}^{\Delta \tau}\right)$ can be replaced by $f_{a_{i}, w}^{\text {in }}\left(\tau+1-t_{a_{i}}^{\Delta \tau}\right)$, i.e., the passengers entering into node $a_{i}$ first reach the walking area. The instantaneous travel time $t_{a_{i}}(\tau+1)$ in the walking area of node $a_{i}$ by time $\tau+1$ is given as

$$
t_{a_{i}}(\tau+1)=\sum_{g=\tau+1-t_{a_{i}}^{\Delta \tau}}^{\tau+1} v_{a_{i}}(g) \cdot \Delta \tau
$$

where $v_{a_{i}}(g)$ is the passenger flow speed of node $a_{i}$ during the time interval $g$ for the group of the passengers referred to by Equation (14). The average passenger flow density in the walking area $k_{a_{i}}(\tau)=n_{a_{i}, w}(\tau) /\left(w_{a_{i}} \cdot l_{a_{i}, w}(\tau)\right)$ will be applied to replace the real density for simplicity.
The flow balance equation of the outflow demand of node $a_{i}$ at time $\tau+1$ can be given as

$$
x_{a_{i}}^{\text {out }}(\tau+1)=x_{a_{i}}^{\text {out }}(\tau)-f_{a_{i}}^{\text {out }}(\tau)+f_{a_{i}, w}^{\text {out }}(\tau)
$$

To obtain the passenger flow speed in the walking area of node $a_{i}$, which needs to be handled in Equation (12), the passenger flow fundamental diagram relating to speed and density is introduced. There exist a variety of fundamental diagrams in the literature $[40,41]$. The following form [42], one of the most widely used in previous literature, is applied to give the speed on different nodes of facilities.

$$
v(k)=v_{f}\left\{1-\exp \left[-\gamma\left(\frac{1}{k}-\frac{1}{k_{\text {jam }}}\right)\right]\right\}, \quad 0 \leq k \leq k_{\text {jam }},
$$

where $v_{f}$ and $k_{\text {jam }}$ are the free-flow speed and the jam density, respectively. $\gamma$ denotes a shape parameter.

The relations between speed and density for facilities with multi-directional flows such as the concourse and the platform need to be specified. By adding the reduction factor to the relations of unidirectional walkways, it can be obtained by

$$
v_{m}(k)=\varphi \cdot v_{w}(k),
$$

where $v_{m}(k)$ is the passenger flow speed of multi-directional facility, $v_{w}(k)$ is the passenger flow speed of unidirectional walkways.

\section{Case Study}

In this section, a case study of simulating passenger flow in a subway station based on system dynamics is proposed. Firstly, the general details of the station are given, and the experiment is carried out accordingly. Secondly, a comparison between the real data and the results obtained by the proposed model 


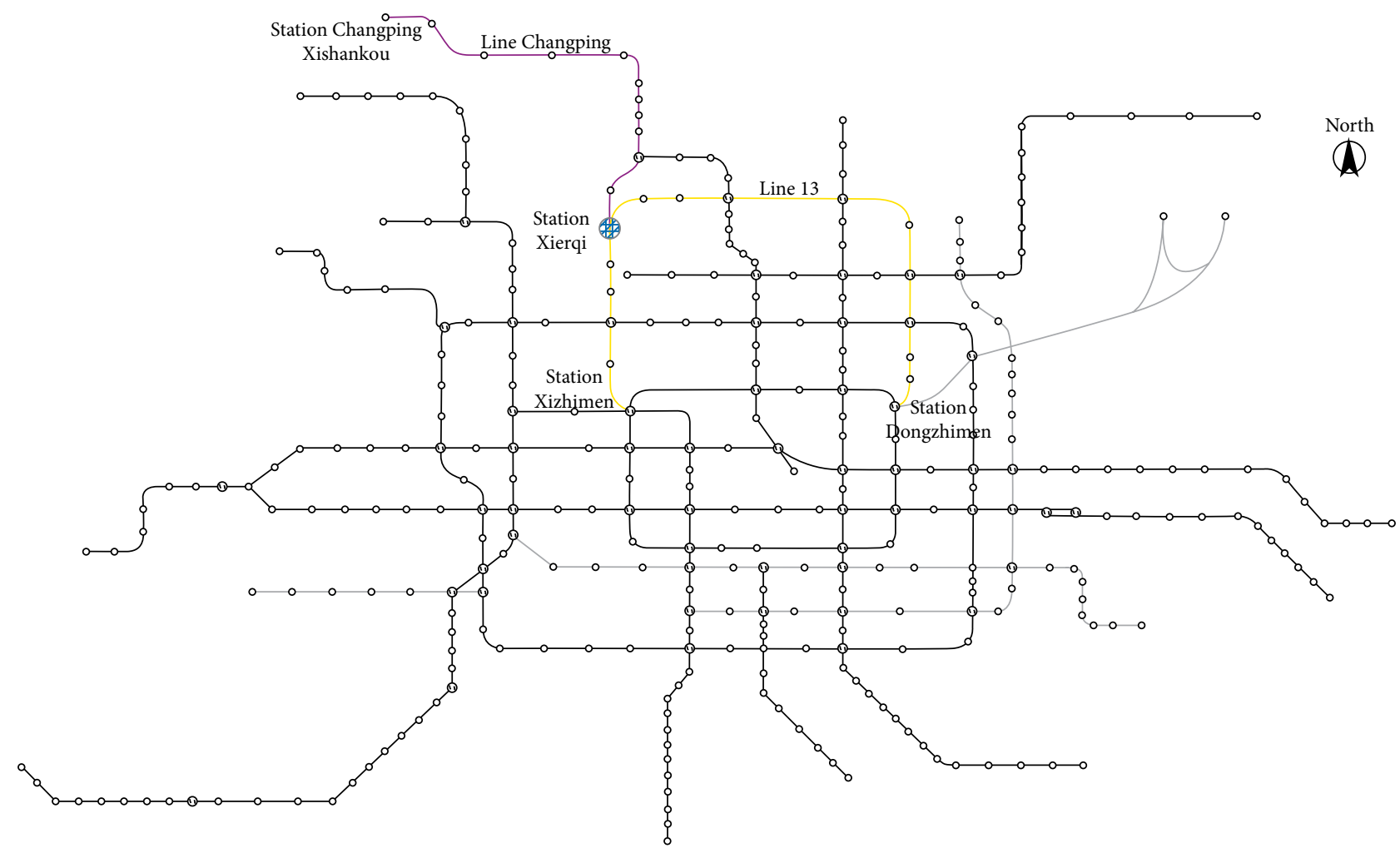

Figure 5: The map of Beijing subway.

is implemented. Finally, some useful applications of the proposed model, including the dynamic change in the number of passengers and the LOS of facilities are presented.

4.1. Station Description. Figure 5 shows the topology of the subway network in Beijing in which the location of the Xierqi station is marked. It serves a large scale of commuting passengers during rush hours, because most commuters live in the suburbs of Beijing, while most of them work at downtown. The Xierqi station is the critical transfer station connecting two important subway lines: the Changping line (CPL) extending to the suburbs and the Line 13 (L13) operating at downtown area, leading to a large number of transferring passengers between them. Besides, it also serves many passengers going outside of the station with lots of industrial states around it. As can be seen from Figure 6, the number of passengers in every half an hour is presented during 7:00 am-9:30 am, where the number of outbound and transferring passengers accounts for $30 \%$ and $47 \%$ of the sum of passengers in the station respectively.

The proposed case study of the Xierqi station, illustrated in Figure 7, has a solely integrated concourse serving for the whole station. The station has two entrances; both of them are bi-directional and locate at both ends of the concourse. The station has the side platforms for both directions of Line 13: UP_L13 heading to the Xizhimen station directly connecting to the concourse, and DP_L13 heading to the Dongzhimen station connecting with the concourse by a series of staircases and escalators. There is only one side platform serving for both directions of CPL referred to as P_CPL. Flow facilities like the staircases and the escalators are set to connect these holding facilities. The headway times and the dwell times for both directions of L13 running by the timetable of the day studied are $164 \mathrm{~s}$ and $30 \mathrm{~s}$, and for CPL are $360 \mathrm{~s}$ and $160 \mathrm{~s}$, respectively. The maximum load factor is less than $120 \%$ as for L13 and around $140 \%$ for CPL during rush hours. Three empty trains run on U_L13 from 7:17 am to 7:33 am in every 8 minutes.

For the overall gathering and scattering process of the whole station, there exist two specific queuing processes: one is the process of leaving the platform, and the other is the process of waiting-boarding trains. They are the representatives of the disordered queuing and ordered queuing respectively. In the following, these two processes will be separately demonstrated by the processes of leaving P_CPL and waiting-boarding trains at UP_L13.

For the process of leaving P_CPL, the whole platform can be divided into two symmetrical parts, and the results will be represented with the north half part within the dot-dash rectangle as shown in Figure 8 considering the following two aspects. Firstly, the physical structure of $\mathrm{P} \_\mathrm{CPL}$, composed of trains of CPL, two groups of G1 and G2 located at both ends of P_CPL, and both E6 and E7 in the middle, is symmetrical in the south-north direction. Secondly, the two symmetrical parts have similar demand of passenger flow, who select one of the two platform exits (G1 or G2) to leave the platform. According to the route choice [43], these two parts have a similar distribution of passengers.

For the process of waiting-boarding trains on UP_L13, we divide the whole platform into two parts. The results will be represented with the north half part within the dot-dash 


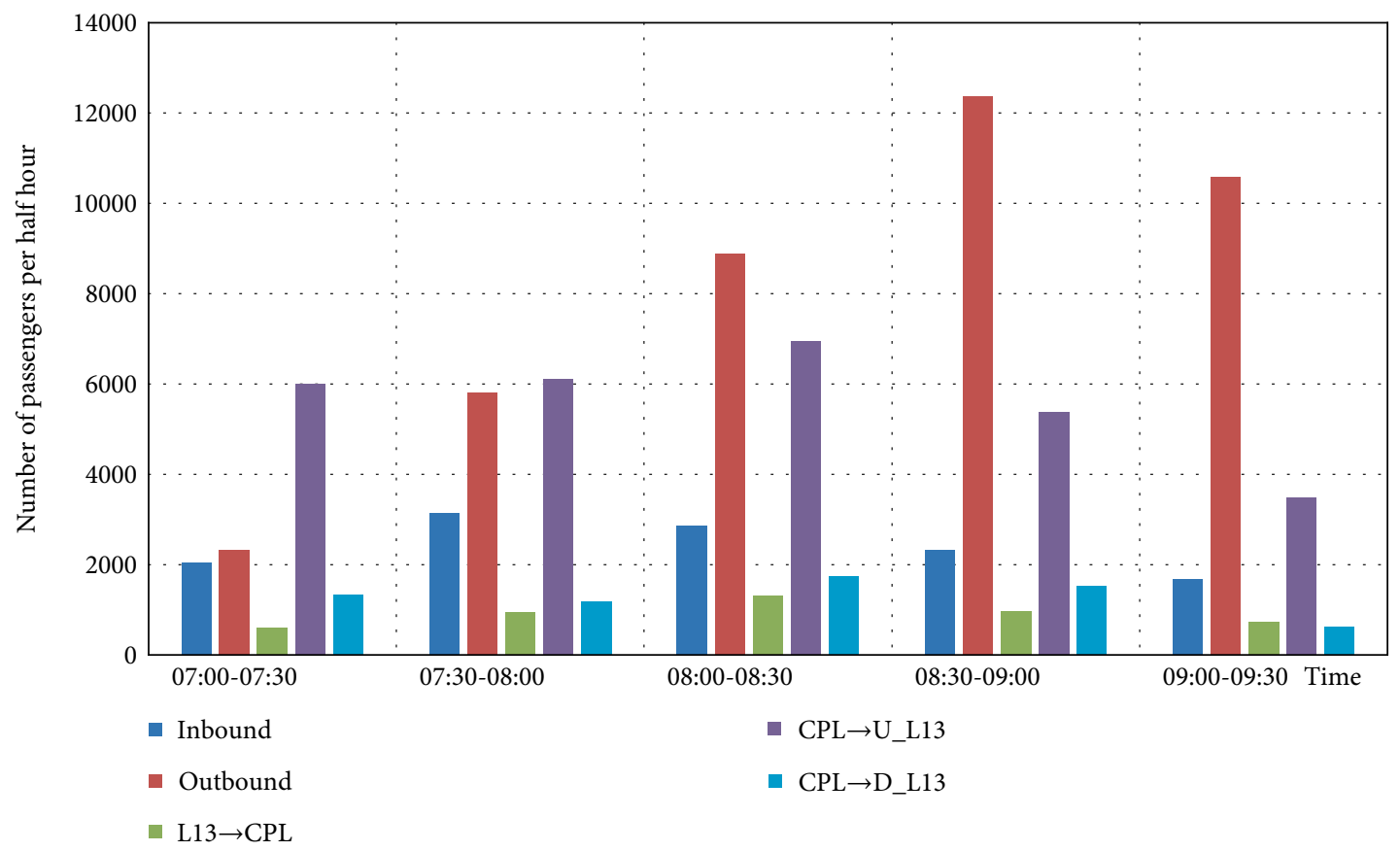

FIgURE 6: Number of passengers per half-hour in Xierqi station.

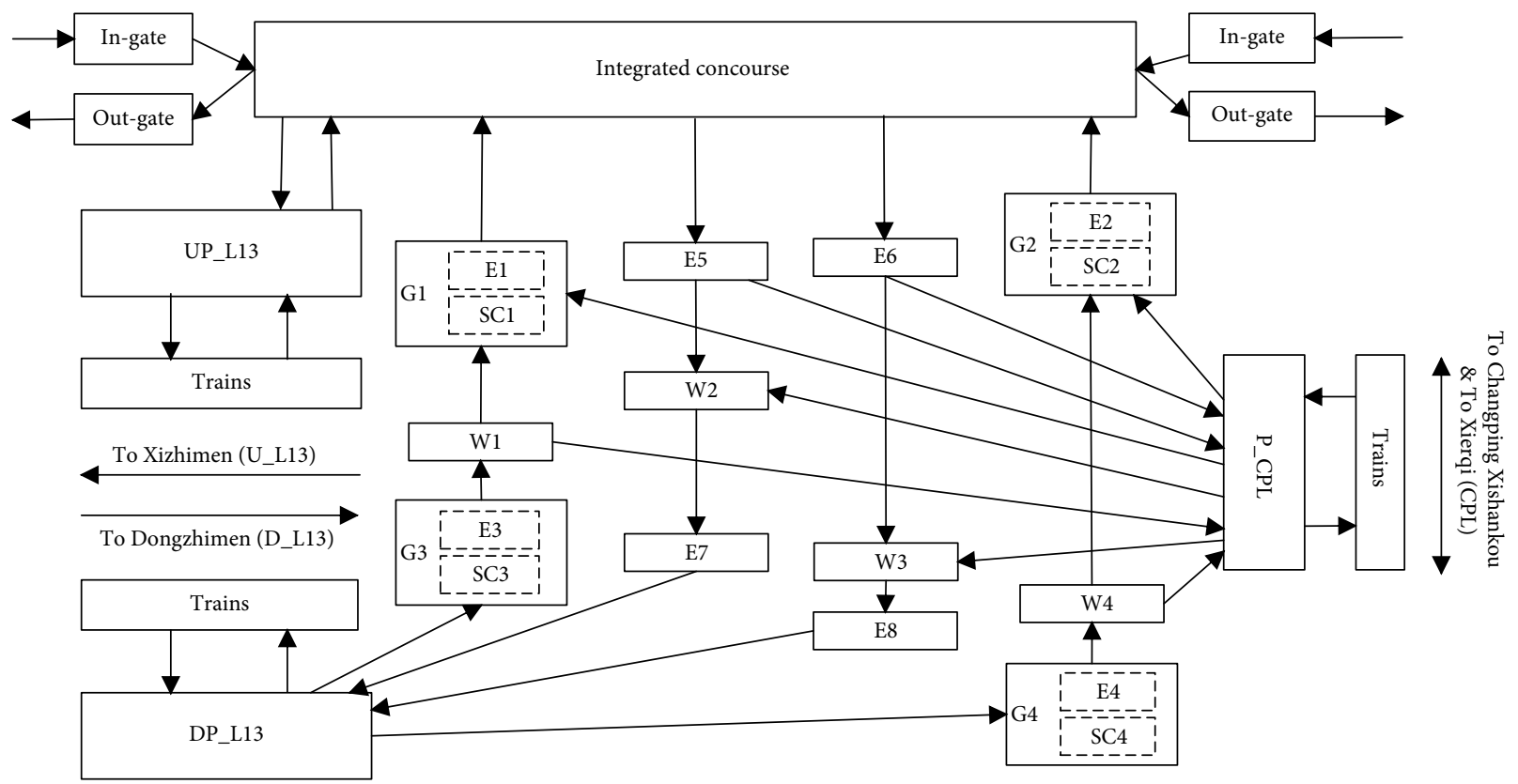

FIGURE 7: Xierqi station network. Abbreviations of facilities will be applied for convenience: Escalator: E, Staircase: SC, Walkway: W, Line 13: L13, Changping line: CPL. The up and down directions of L13 are called as U_L13 and D_L13 respectively. The separate side platforms for them are referred to as UP_L13 and DP_L13. The platform for CPL is P_CPL.

rectangle, as shown in Figure 9 according to the following two aspects. Firstly, the two parts including five subareas are symmetrical, and the concourse and in or out gate machines along with it are symmetrical. Besides, the two symmetrical parts have a similar demand of passenger flow, such as the passengers entering the station by in-gate $A$ and in-gate $B$, the passengers leaving the station by out-gate $A$ and out-gate $B$ respectively. According to the route choice [43], these two parts have a similar distribution of passengers.

4.2. Model Specification and Calibration. A sample of paired data between speed and density is obtained by field investigation. The parameters relating to speed and density are provided in Table 1 after fitting the sample data for a $95 \%$ 


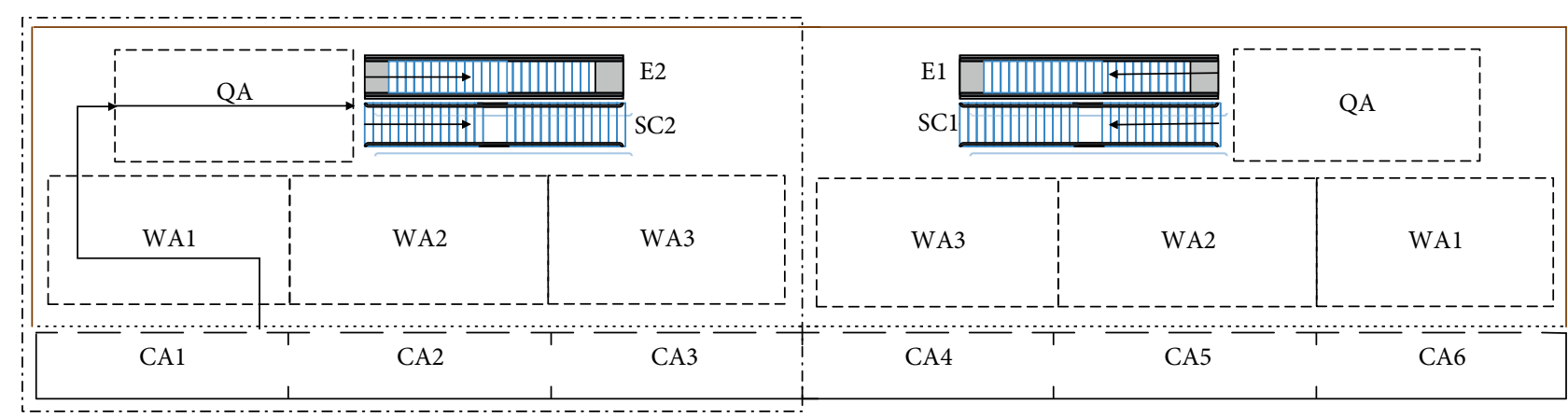

FIgURE 8: Area division for P_CPL. The queuing area, the walking area, and the carriage marked as QA, WA, and CA respectively. WA is divided into three parts corresponding to CAs. A representative of the walking path on P_CPL starting from one of the train doors is shown in solid black arrows.

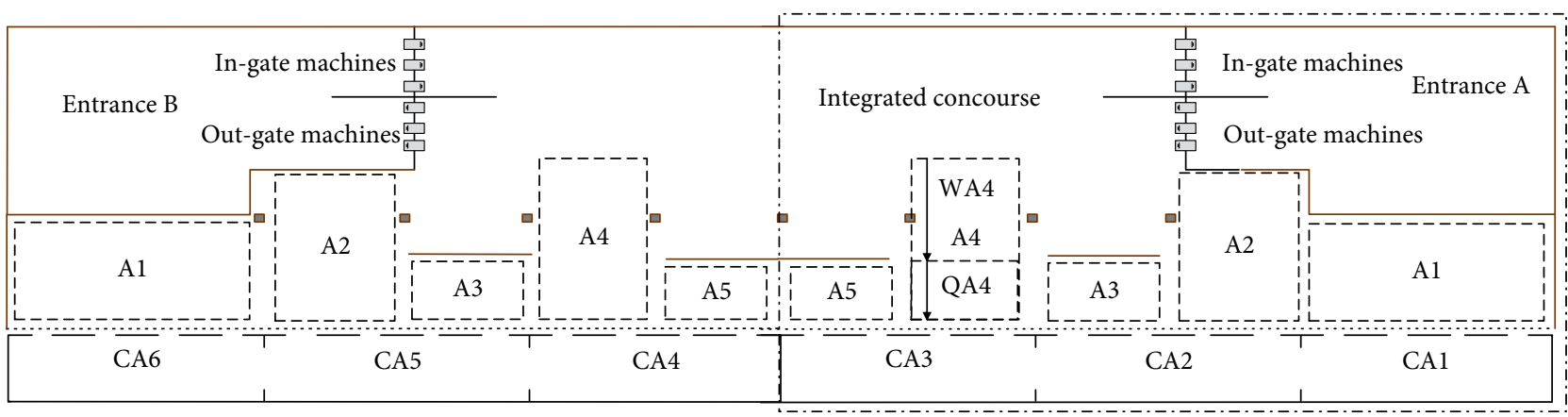

FIGURE 9: Area division of UP_L13. Either part of UP_L13 can be divided into five subareas referred to as A1-A5. For the process of waitingboarding trains, each sub-area consists of the queuing area and walking area marked as QA and WA respectively, as illustrated by A4 including WA4 and QA4. A representative of a path along the UP_L13 is shown in solid black arrows.

TABLE 1: Flow facilities and their parameters for fundamental diagrams.

\begin{tabular}{lcccccccc}
\hline \multirow{2}{*}{ Facility name } & \multirow{2}{*}{ Number } & Direction & Length $(\mathrm{m})$ & Width $(\mathrm{m})$ & Height $(\mathrm{m})$ & \multicolumn{3}{c}{ Parameters relating speed and density } \\
& & & & & & $v_{f}$ & \multicolumn{1}{c}{$k_{\text {jam }}$} \\
\hline Staircase & 2 & Up & 19 & 2 & 7.2 & 0.8095 & 1.799 & 4.827 \\
Walkway & 4 & Single & 19.5 & 7 & - & 1.364 & 1.412 & 4.891 \\
Staircase & 2 & Down & 19 & 2 & 7.2 & 0.8874 & 1.641 & 4.926 \\
\hline
\end{tabular}

confidence interval. Route choice proportion corresponding to $\delta_{a_{i} \rightarrow b_{j}}(\tau)$ in Equation (3) is another type of parameter need to be confirmed. All of these proportions adopt 0.5 through field investigation and referring to the route choice [43].

The reduction factor $\varphi$ for the concourse is estimated at 0.85 and that for the platform at 0.75 by statistical analysis of sample data. Besides, the parameters related to sub-areas of UP_L13 are shown in Table $2 . \Delta l$ the average distance between two adjacent passengers is a random variable and follows a uniform distribution for a specific situation of queuing. Generally, it is inversely proportional to the ratio of the number of queuing passengers to the size of maximum space. Based on field investigation and referring to [44], the values of $\Delta l$ for subareas of UP_L13 are set between $0.3 \mathrm{~m}$ and $0.4 \mathrm{~m}$. It also shows the allocation proportions for passengers selecting different subareas of UP_L13. The LOS evaluation standard for subway platform can be found in Figure 10 [45].
TABLE 2: Parameters for subareas of UP_L13.

\begin{tabular}{lccccc}
\hline Subareas of UP_L13 & A1 & A2 & A3 & A4 & A5 \\
\hline$\Delta l(\mathrm{~m})$ & 0.35 & 0.4 & 0.3 & 0.4 & 0.3 \\
$\begin{array}{l}\text { Allocation proportion } \\
\begin{array}{l}\text { Number of queues for each } \\
\text { train door }\end{array}\end{array}$ & 0.3 & 0.2 & 0.15 & 0.2 & 0.15 \\
\hline
\end{tabular}

4.3. Model Building in the Simulation Platform. The visualized modeling is carried out based on the library of system dynamics in AnyLogic that is developed on Java. The elements used to build the stock-flow diagram and their implications are given as follows.

We build the stock-flow diagram for the process of leaving P_CPL, as shown in Figure 11, based on the platform layout, as shown in Figure 8 and characteristics of passenger flow. First, a group of passengers alighting from the trains walk on 


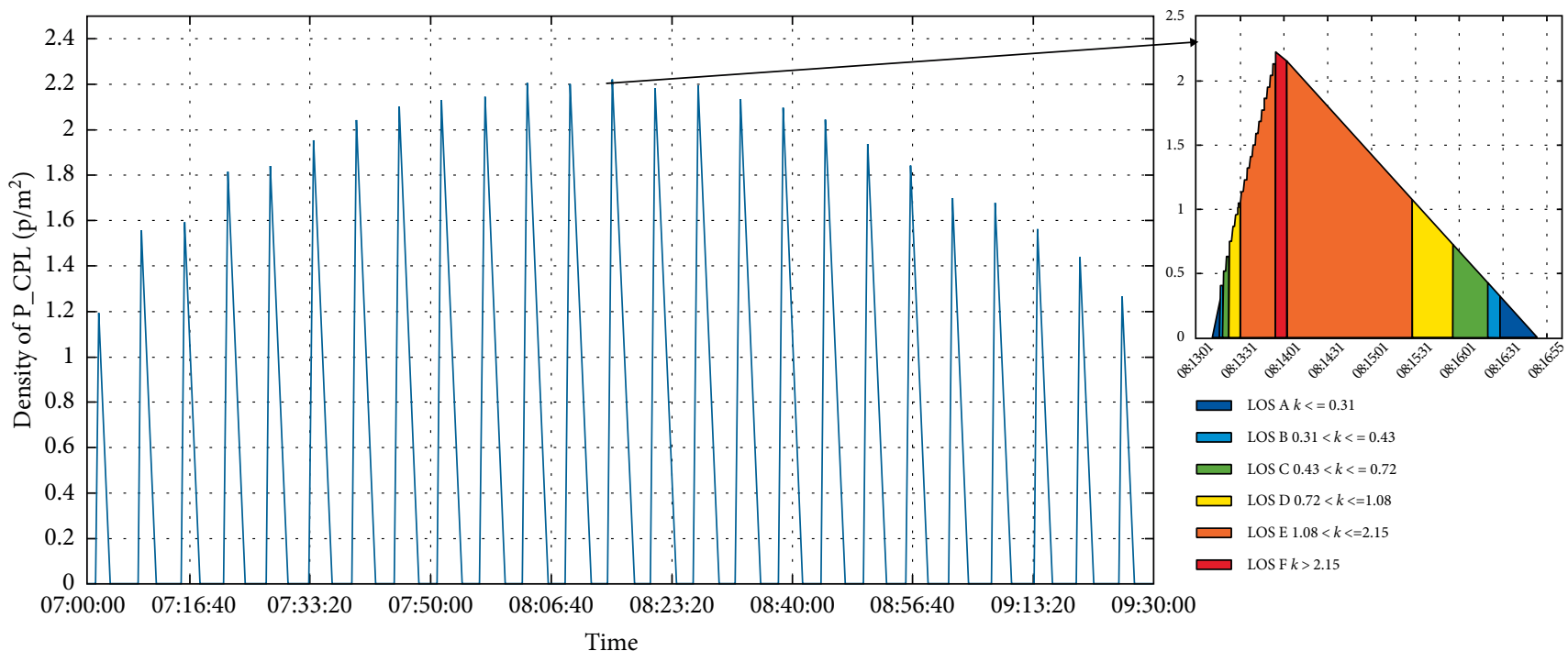

FIgURE 10: Time-varying LOS on P_CPL for the full simulation period.

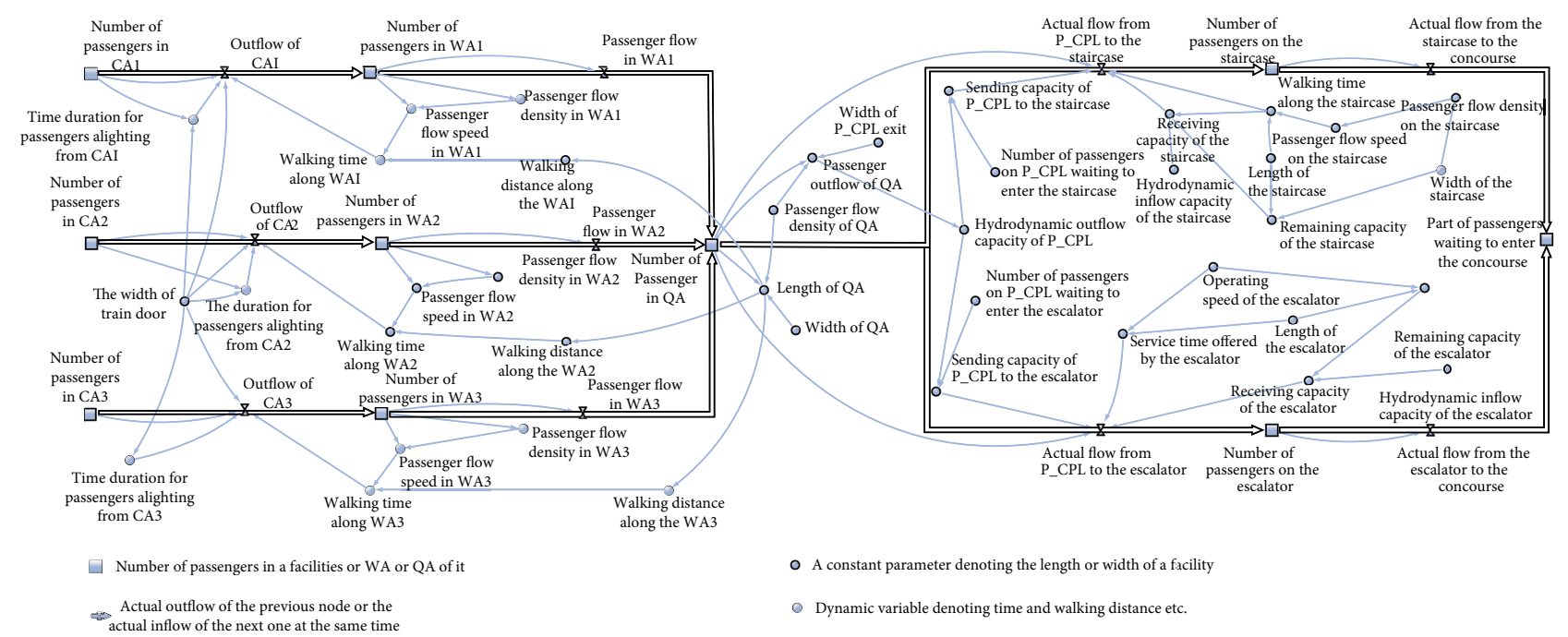

FIGURE 11: Stock-flow diagram for leaving P_CPL.

WA and then aggregate at QA waiting to enter the next facility: staircase or escalator. It has a one-to-many relation between the P_CPL and the downstream nodes, including the staircase $\mathrm{SC} 2$ and the escalator E2.

4.4. Comparison with Real Data. Figure 13 shows a histogram of the number of passengers at QA of P_CPL during one cycle which is the time period between the arrival time of the first train and that of the next referencing [26] to get detailed information of the cycle), according to the real data and as obtained by the proposed model. The proposed model performs fairly well by comparing the results of the proposed model referred to as predicted values with the real data. According to the difference between predicted values and the real data, this process can be divided into two phases: the predicted values are less than the real data at first phase, and the predicted values are larger than the real data at the second phase. From the field observation, we learn that for passengers in the first phase tend to walk faster on P_CPL than average speed. While for passengers in the second phase, they walk slower than average speed and not hurry to reach the exit of P_CPL where there has been a lot of passengers. For the same reason, the start time of queuing, when the first passenger alighting from any train door reaches QA, obtained by real investigation (12 s), is less than that computed by the proposed model (15 s).

Figure 14 shows a histogram of the number of passengers stranded at UP_L13, according to the real data and as obtained by the proposed model. It verifies that the proposed model showing a reasonable agreement with the real data for the process of waiting-boarding trains.

4.5. The Dynamic Change in the Number of Passengers. Xierqi station has the terminal station of CPL connecting with L13, CPL trains stopped at this station in rush hours have higher load factors since they carry a lot of passengers having boarded 


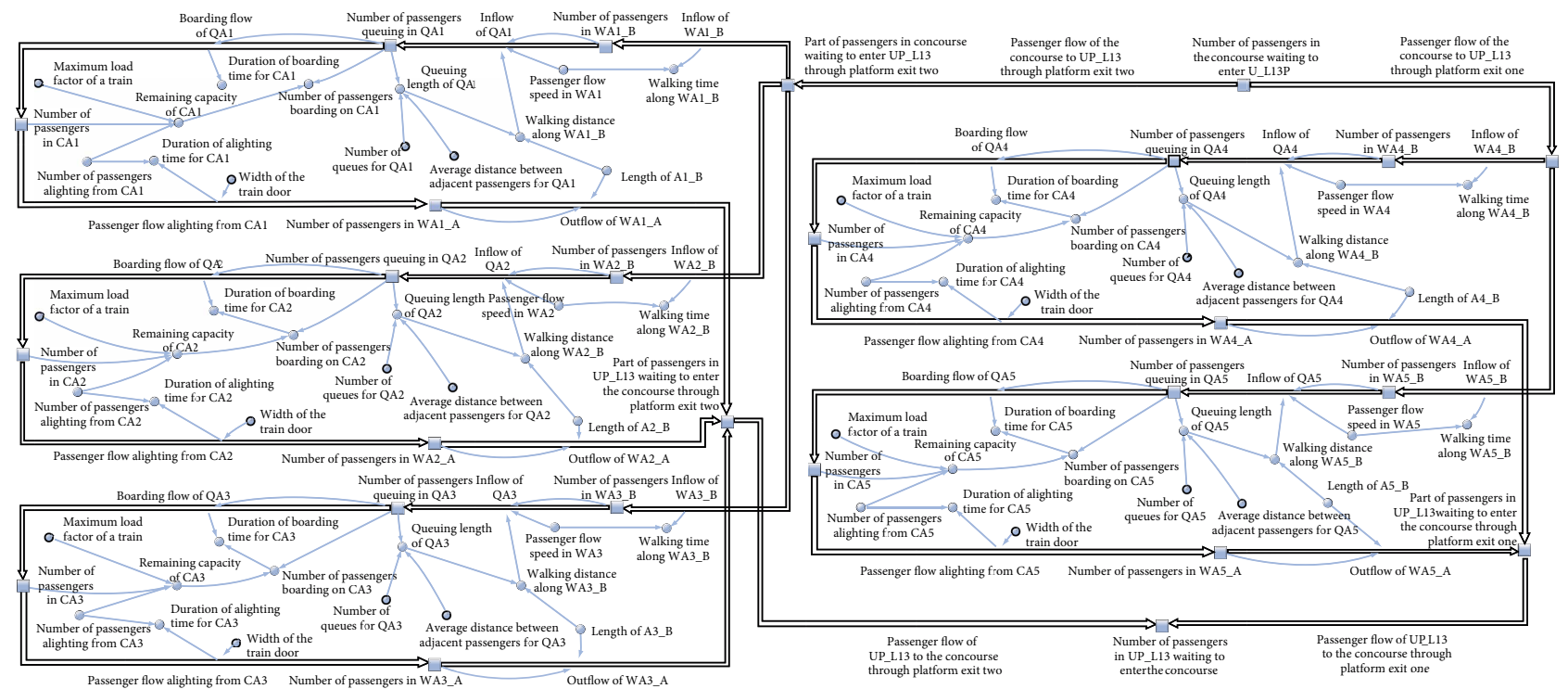

FIGURE 12: Stock and flow diagram for waiting-boarding trains at UP_L13. To differ from the alighting process where WA is marked as WA_B, and there is no QA in this process. WA for the process of waiting-boarding trains is marked as WA_A.

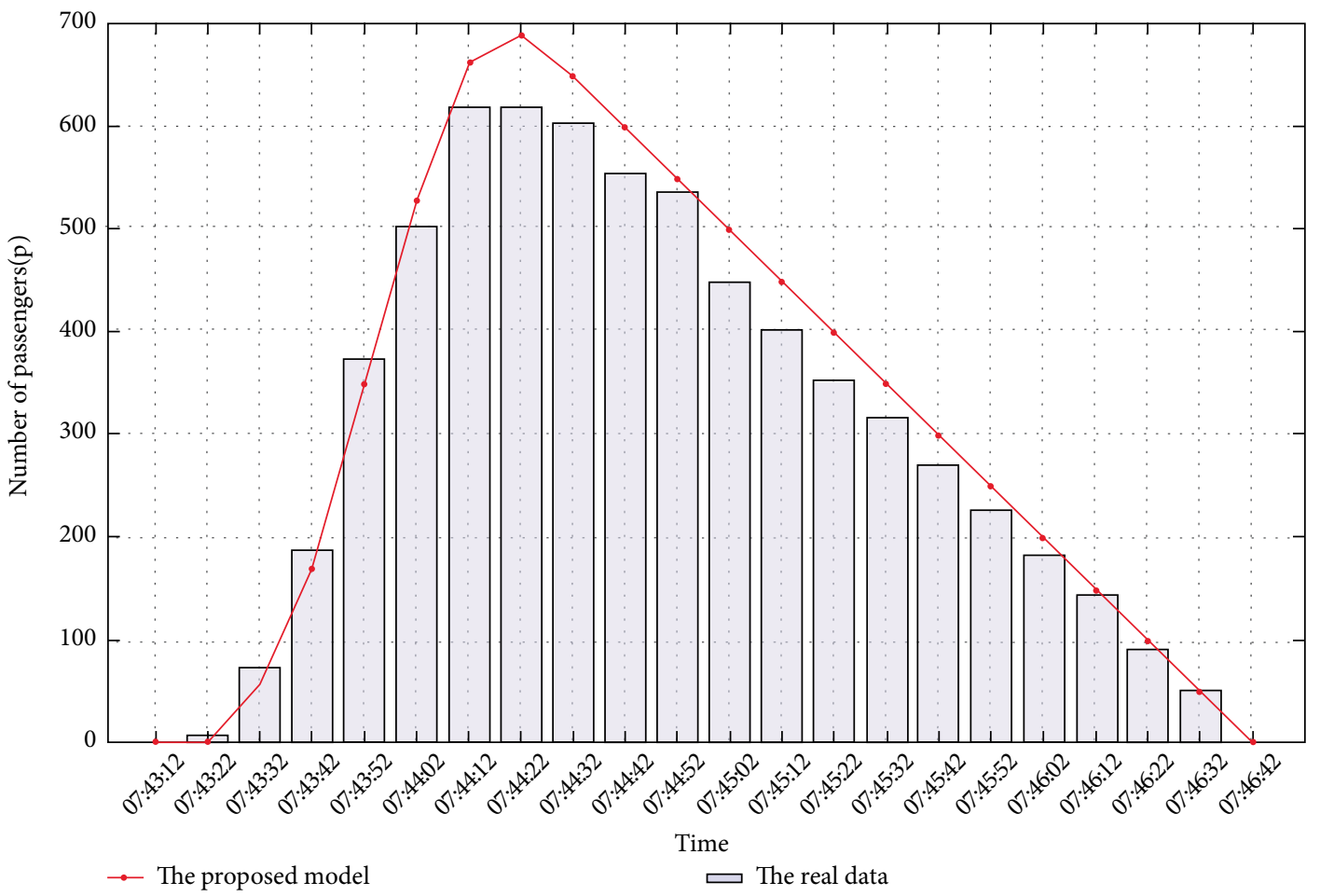

FIGURE 13: The number of passengers for the process of leaving P_CPL.

on the trains at previous stops. The large scale of passengers alighting from these trains first walk through P_CPL, and then get into transferring passageway G2 including E2 and SC2. Figure 15 presents the number of passengers alighting from CPL trains and that queuing at the exit of P_CPL over the whole simulation time. The platform suffers from a large scale of passengers over 550 each cycle from 7:20 am to 9:20 am, especially more than 700 passengers from 7:50 am to 8:30 am.
The peak value of the alighting passengers is higher than that of queuing, mainly due to the difference between the outflow of CA and the outflow of P_CPL. Another reason is that part of the CPL alighting passengers around 10\% transferring to DP-L13 via W2 and E7 as shown in Figure 6.

Figure 16(a) provides a dynamic change in the number of passengers waiting on UP_L13. The platform has an aggregation of passengers nearly over 1000 from 8:04 am to 8:48 am 


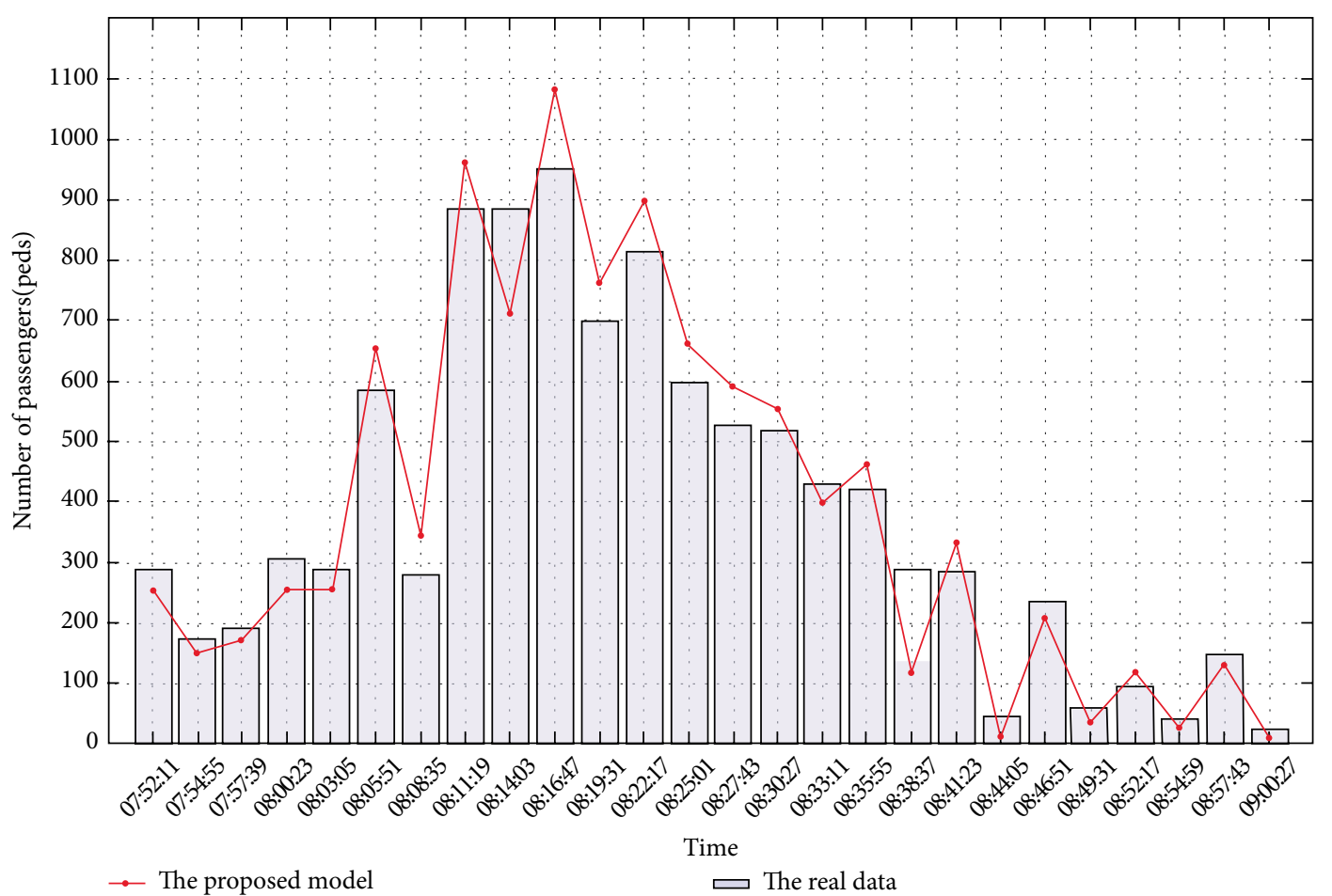

FIGURE 14: The number of stranded passengers for the process of waiting-boarding trains at UP_L13.

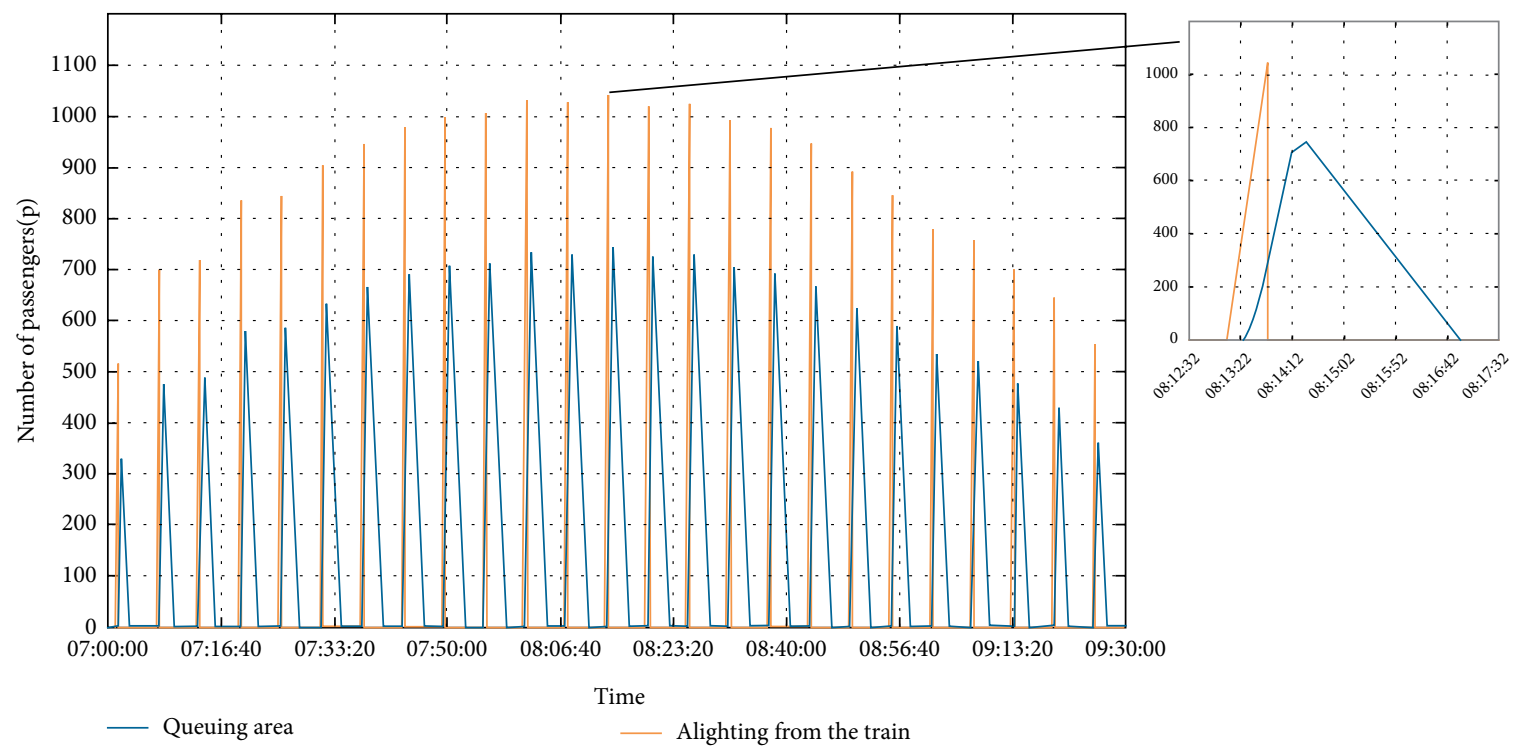

FIGURE 15: The number of passengers queuing at the exit of P_CPL over the whole simulation time.

and reaches the maximum at 8:24 am. The passengers are composed of two parts: one is the discrete but large transferring passengers coming from $\mathrm{CPL}$, and the other is the continuous but small inbound passengers. In the period of one cycle when there is no train stopping at the station, if there are no transferring passengers, the number of passengers on UP_L13 increase slowly; otherwise it rose sharply. In the period of one cycle when a train stop at the station, the number of passengers on UP_L13 drops off quickly since the flow into CAs (the outflow of UP_L13) is rather larger than the inflow of UP_L13. Since the headway times of CPL is more than twice as much as UP_CPL, continuous declines in successive two cycles may arise. It also can be known that subareas with approximately same size have a similar change in the number of passengers as illustrated in Figures 16(b), 16(d), and 16(f) for a smaller size, and Figures 16(c) and 16(e) for a larger size.

Figure 10 shows the change in the average density of $\mathrm{P}_{-}$ CPL over the whole simulation period according to the density-based classification of the LOS in Transit Capacity and Quality of Service Manual [42]. The LOS during each cycle 


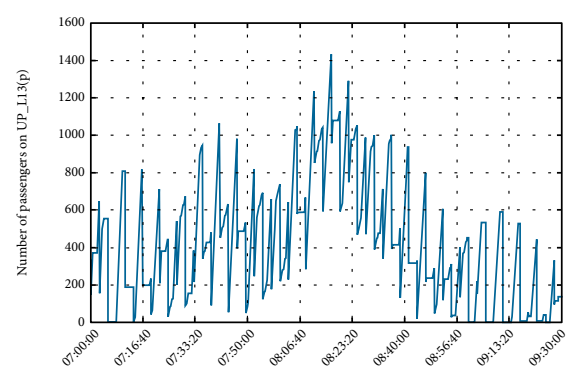

Time

(a)

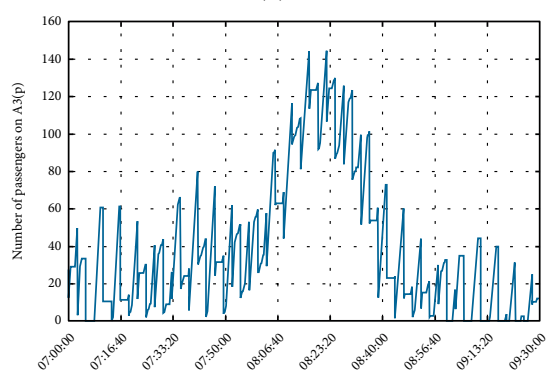

d)

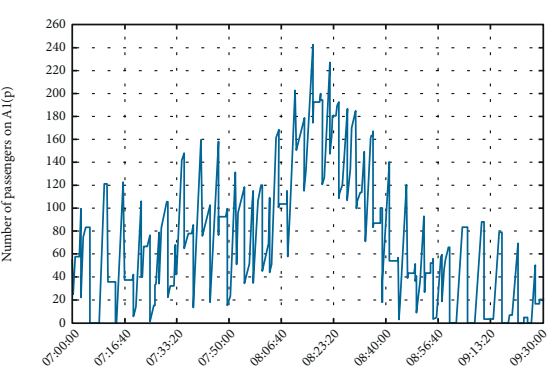

Time

(b)

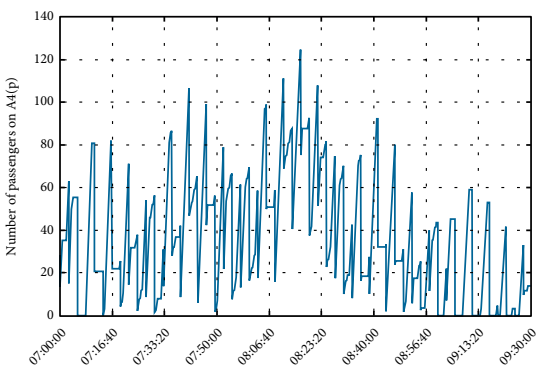

(e)

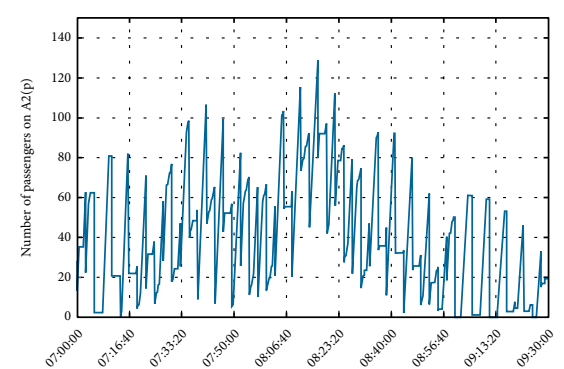

Time

(c)

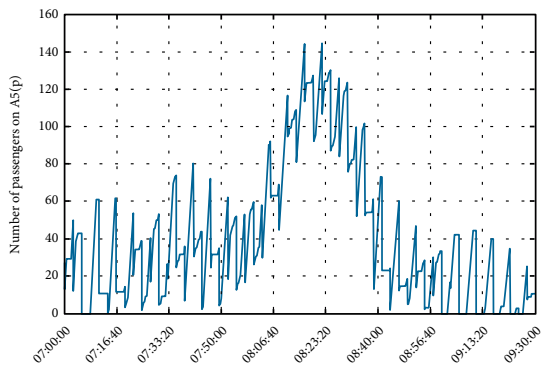

Time

(f)

FIGURE 16: The change in the number of passengers of Line 13 and its subarea for the full simulation period. (a) UP_L13, (b) A1, (c) A2, (d) A3, (e) A4, and (f) A5.

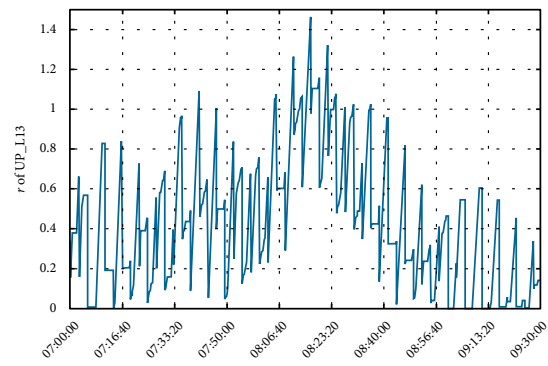

Time

(a)

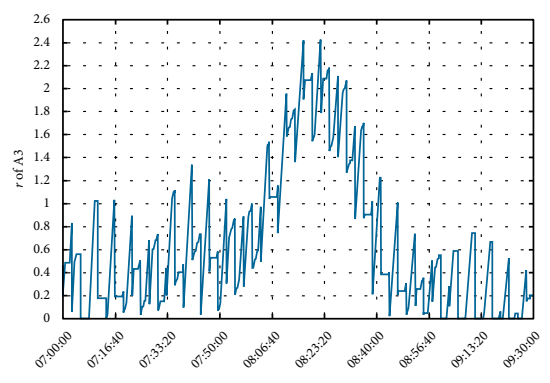

(d)

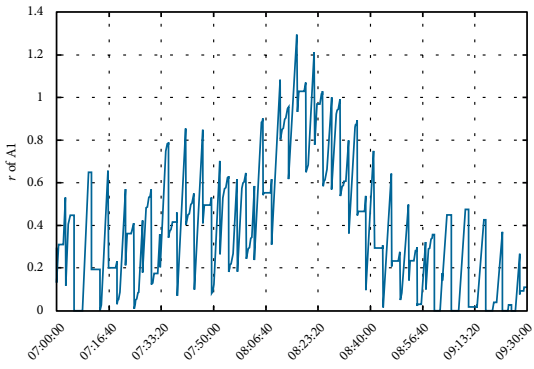

(b)

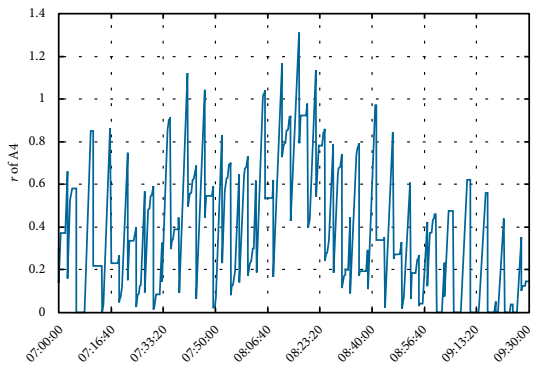

(e)

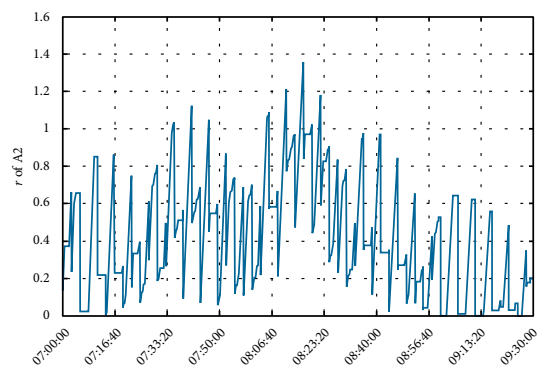

Time

(c)

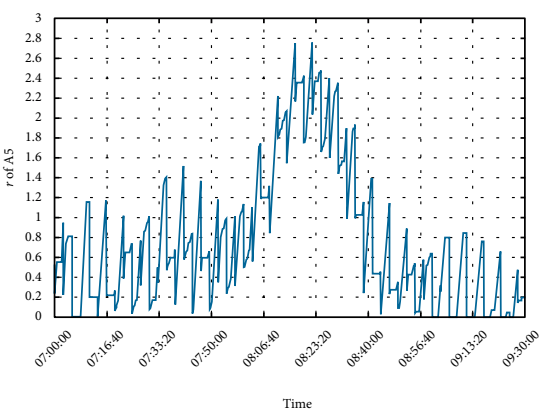

(f)

FIGURE 17: $r$ for UP_L13 and its subareas (a) UP_L13, (b) A1, (c) A2, (d) A3, (e) A4, and (f) A5.

changes greatly, as shown in Figure 10. The LOS of P_CPL for one cycle can be divided into two phases, as the change in the number of passengers in Figure 15. At the first phase, the LOS on P_CPL changes rapidly from LOS A to LOS F since the rapid increase of passengers on P_CPL. At the second phase, when the inflow is less than the outflow, the LOS of P_CPL changes gradually from LOS F to LOS A. The time in the congested status of the whole platform including LOS E and LOS F approximately accounts for $30 \%$ of one cycle.

From the field observation, it can be known that passengers waiting for trains queue orderly at UP_L13, so we set $r$, the ratio of queue length to the total physical length of the platform, 
as the index to measure the status of the platform. $r$ can be an index greater than one to present the difference between holding capacity and the required space for holding passengers. Two assumptions are made as follows: (1) the preferred maximum queuing space $\Delta l$ for passengers on the platform in Equation (11) will be set as $0.35 \mathrm{~m}$, as shown in Table 2; (2) the standard number of queues is two. However, more than two queues can be found in the real world. The following two aspects explain why $r$ will be greater than one. First, integrated concourse can hold some passengers coming from the spillback of passengers queuing at UP_L13, allowing for its connection with the concourse in regard to QA_2 and QA_5, as shown in Figure 9. In addition, as the above second assumption, more queues will be formed under extreme space limitation.

As shown in Figure 17(a), $r$ is greater than one from 8:10 am to $8: 45 \mathrm{am}$, which means that for the whole platform, the demand of passengers is larger than the holding capacity. It shows that extra space or more queues are in need. A detailed analysis can be given to subareas. Through field investigation, it is observed that the excessive passengers in A2 and A4 (see Figures $17(\mathrm{c})$ and $17(\mathrm{e})$ ), will wait at the concourse directly connecting with the platform, having an influence on movements of other passengers walking on the concourse. For A3 and A5 (see Figures 17(d) and 17(f)), where the queuing space is severely restricted, passengers self-organize with multiple queues ( 4 queues can be found by field investigation) or reduce $\Delta l$, which increase passengers' body contact and reduce the LOS. QA1 (see Figure 17(b)) not directly connecting with the concourse and is a little insufficient in space. It can be found that some passengers aggregate at the tail of the queues.

\section{Conclusion}

A macroscopic flow simulation model based on system dynamics has been developed. It offers a framework of simulating the passenger flow in the gathering and scattering process for stations with complicated layouts. All the elements of the five key factors are incorporated into the casual-loop diagram. From the aggregation level, the dynamic change in passengers' status could be presented in a rather reasonable way, especially for high-density passenger flows with characteristics of fluid dynamics. It figures out the passenger flow transmission between nodes and passenger flow movement on facilities while considering the queuing due to the capacity constraint. The disordered and ordered queuing are demonstrated by the process of leaving the platform and that of waiting-boarding the trains respectively in the case study, showing that the proposed model performs well.

In the future, the developed framework may be improved and extended in several ways. First, the proportion of the route choices for passengers may be formulated in a universal way, instead just suitable for the station studied in this paper. Second, more statistical indicators such as the walking time of paths would allow reinforcing its application in the real world. Third, the proposed model could be extended to be a simulation tool for the supervision of the passengers' status in a station or employed in implementing passenger flow control.

\section{Data Availability}

The data used to support to the findings of this study have not been made available because some of them are the internal operation data by the Beijing subway, the author was not granted the right to disclose.

\section{Conflicts of Interest}

The authors declare that they have no conflicts of interest regarding the publication of this paper.

\section{Acknowledgments}

This work was supported by the National Key R\&D Program of China (2016YFB1200402).

\section{References}

[1] W. H. K., Lam, C.-Y. Cheung, and C. F. Lam, "A study of crowding effects at the Hong Kong light rail transit stations," Transportation Research Part A, vol. 33, no. 5, pp. 401-415, 1999.

[2] D. King, S. Srikukenthiran, and A. Shalaby, "Using simulation to analyze crowd congestion and mitigation at canadian subway interchanges:case of bloor-yonge station, Toronto, Ontario," Transportation Research Record: Journal of the Transportation Research Board, vol. 2417, no. 1, pp. 27-36, 2014.

[3] C. S. Jiang, Y. F. Deng, C. Hu, H. Ding, and W. K. Chow, "Crowding in platform staircases of a subway station in China during rush hours," Safety Science, vol. 47, no. 7, pp. 931-938, 2009.

[4] L. Huang, T. Chen, Y. Wang, and H. Yuan, "Congestion detection of pedestrians using the velocity entropy: a case study of love parade 2010 disaster," Physica A Statistical Mechanics \& Its Applications, vol. 440, pp. 200-209, 2015.

[5] X. Chen, H. Li, J. Miao, S. Jiang, and X. Jiang, "A multiagentbased model for pedestrian simulation in subway stations," Simulation Modelling Practice and Theory, vol. 71, pp. 134-148, 2017.

[6] A. Kirchner, H. Klupfel, K. Nishinari, A. Schadschneider, and M. Schreckenberg, "Discretization effects and the influence of walking speed in cellular automata models for pedestrian dynamics," Journal of Statistical Mechanics Theory and Experiment, vol. 2004, no. 10, p. P10011, 2004.

[7] D. Helbing, I. J. Farkas, and T. Vicsek, "Simulating dynamical features of escape panic," Nature, vol. 407, no. 6803, pp. 487-490, 2000.

[8] M. Moussaid, D. Helbing, and G. Theraulaz, "How simple rules determine pedestrian behavior and crowd disasters," PNAS, vol. 108, pp. 6884-6888, 2011.

[9] A. Treuille, S. Cooper, and Z. Popovic, "Continuum crowds," ACM Transactions on Graphics, vol. 25, no. 3, pp. 1160-1168, 2006.

[10] C. F. Daganzo, "The cell transmission model: a dynamic representation of highway traffic consistent with the hydrodynamic theory," Transportation Research Part B, vol. 28, no. 4, pp. 269-287, 1994. 
[11] C. F. Daganzo, “The cell transmission model, part II: network traffic," Transportation Research Part B, vol. 29, no. 2, pp. 79-93, 1995.

[12] G. Flötteröd and C. Osorio, "Stochastic network link transmission model," Transportation Research Part B, vol. 102, pp. 180-209, 2017.

[13] Y. Jiang, L. H. Juanxiu Zhu, X. Lin, and A. Khattak, "A G/G(n)/ $\mathrm{C} / \mathrm{C}$ state-dependent simulation model for metro station corridor width design," Journal of Advanced Transportation, vol. 50, no. 3, pp. 273-295, 2016.

[14] I. E. Manataki and K. G. Zografos, "A generic system dynamics based tool for airport terminal performance analysis," Transportation Research Part C: Emerging Technologies, vol. 17, no. 4, pp. 428-443, 2009.

[15] Q. Yunchao, Z. Gao, Y. Xiao, and X. Li, "Modeling the pedestrian's movement and simulating evacuation dynamics on stairs," Safety Science, vol. 70, pp. 189-201, 2014.

[16] Z. Qi, H. Baoming, and L. Dewei, "Modeling and simulation of passenger alighting and boarding movement in Beijing metro stations," Transportation Research Part C, vol. 16, no. 5, pp. 635-649, 2008.

[17] V. J. Blue and J. L. Adler, "Cellular automata microsimulation for modeling bi-directional pedestrian walkways," Transportation Research Part B: Methodological, vol. 35, no. 3, pp. 293-312, 2001.

[18] G. Flötteröd and G. Lämmel, "Bidirectional pedestrian fundamental diagram," Transportation Research Part B, vol. 71, pp. 194-212, 2015.

[19] M. Nasir, C. P. Lim, S. Nahavandi, and D. Creighton, "A genetic fuzzy system to model pedestrian walking path in a built environment," Simulation Modelling Practice and Theory, vol. 45, pp. 18-34, 2014.

[20] F. S. Hänseler, M. Bierlaire, B. Farooq, and T. Mühlematter, "A macroscopic loading model for time-varying pedestrian flows in public walking areas," Transportation Research Part B: Methodological, vol. 69, pp. 60-80, 2014.

[21] M. P. H. Raadsen, M. C. J. Bliemer, and M. G. H. Bell, “An efficient and exact event-based algorithm for solving simplified first order dynamic network loading problems in continuous time," Transportation Research Part B, vol. 92, pp. 191-210, 2016.

[22] W. Himpe, R. Corthout, and M. J. C. Tampère, "An efficient iterative link transmission model," Transportation Research Part B: Methodological, vol. 92, pp. 170-190, 2016.

[23] K. Rahman, N. A. Ghani, A. A. Kamil, A. Mustafa, and M. A. K. Chowdhury, "Modelling pedestrian travel time and the design of facilities: a queuing approach," PloS One, vol. 8, no. 5, p. e63503, 2013.

[24] H. Lu, Y. Jiang, J. Zhu, and Y. Chen, "A PH/PH (n)/C/C statedependent queuing model for metro station corridor width design," European Journal of Operational Research, vol. 240, pp. 109-126, 2015.

[25] I. E. Manatak and K. G. Zografos, "A generic system dynamics based tool for airport terminal performance analysis," Transportation Research Part C, vol. 17, pp. 428-443, 2009.

[26] X. Y. Xu, J. Liu, H. Y. Li, and J.-Q. Hu, "Analysis of subway station capacity with the use of queueing theory," Transportation Research Part C: Emerging Technologies, vol. 38, pp. 28-43, 2014.

[27] R. L. Hughes, "A continuum theory for the flow of pedestrians," Transportation Research Part B, vol. 36, no. 6, pp. 507-535, 2002.

[28] Y. Xia, S. C. Wong, and C. W. Shu, "Dynamic continuum pedestrian flow model with memory effect," Physical Review E, vol. 79, no. 6, p. 066113, 2009.

[29] D. C. Duives, W. Daamen, and S. P. Hoogendoorn, "Continuum modelling of pedestrian flows-Part 2: sensitivity analysis featuring crowd movement phenomena," Physica A Statistical Mechanics \& Its Applications, vol. 447, pp. 36-48, 2016.

[30] F. Xue, W.-N. Fang, and B. Guo, "Rail transit station passenger flow evolution algorithm based on system dynamics," Journal of the China Railway Society, vol. 36, no. 2, pp. 1-10, 2014.

[31] D. C. Duives, W. Daamen, and S. P. Hoogendoorn, "State-of-theart crowd motion simulation models," Transportation Research Part C: Emerging Technologies, vol. 37, pp. 193-209, 2013.

[32] S. P. Hoogendoorn, W. Daamen, V. L. Knoop, J. Steenbakkers, and M. Sarvi, "Macroscopic fundamental diagram for pedestrian networks: theory and applications," Transportation Research Part C: Emerging Technologies, vol. 94, pp. 172-184, 2018.

[33] Y. Li and J. Li, "Multi-class dynamic network traffic flow propagation model with physical queues," Frontiers of Engineering Management, vol. 4, no. 4, pp. 399-407, 2017.

[34] G.-Y. Wu, S.-W. Chien, and Y.-T. Huang, "Modeling the occupant evacuation of the mass rapid transit station using the control volume model," Building and Environment, vol. 45, no. 10, pp. 2280-2288, 2010.

[35] R. M. S. Costa and P. Pavone, "Diachronic biodiversity analysis of a metropolitan area in the mediterranean region," Acta Horticulturae, vol. 1215, pp. 49-52, 2018.

[36] C. Bianca, F. Pappalardo, S. Motta, and M. A. Ragusa, "Persistence analysis in a Kolmogorov-type model for cancerimmune system competition," AIP Conference Proceedings, vol. 1558, pp. 1797-1800, 2013.

[37] M. Asano, A. Sumalee, M. Kuwahara, and S. Tanaka, "Dynamic cell transmission-based pedestrian model with multidirectional flows and strategic route choices," Transportation Research Record: Journal of the Transportation Research Board, vol. 2039, no. 1, pp. 42-49, 2007.

[38] M. Kuwahara and T. Akamatsu, "Dynamic user optimal assignment with physical queues for a many-to-many OD pattern," Transportation Research Part B, vol. 35, no. 5, pp. 461-479, 2001.

[39] X. (Jeff) Ban, J.-S. Pang, H. X. Liu, and R. Ma, "Continuoustime point-queue models in dynamic network loading," Transportation Research Part B: Methodological, vol. 46, no. 3, pp. 360-380, 2012.

[40] A. Seyfried, B. Steffen, W. Klingsch, and M. Boltes, "The fundamental diagram of pedestrian movement revisited," Journal of Statistical Mechanics: Theory and Experiment, vol. 10, no. 10, pp. P10002-P10002, 2005.

[41] M. Nikolić, M. Bierlaire, and B. Farooq, "Probabilistic speeddensity relationship for pedestrians based on data driven space and time representation," Proceedings of the 14th Swiss Transport Research Conference, 2014.

[42] U. Weidmann, Transport Technology of Pedestrians: Transport Characteristics of Pedestrian Traffic, Literature Analysis. IVT Series, Institute for Transport Planning and Systems, ETH Zürich, vol. 90, 1993.

[43] S. P. Hoogendoorn and P. H. Bovy, "Pedestrian route-choice and activity scheduling theory and models," Transportation Research Part B, vol. 38, no. 2, pp. 169-190, 2004.

[44] Group and Y. Liu, "Editorial board of traffic engineering manual of China highway and transportation society," Traffic Engineering Manual, China Communications Press, Beijing, 1998.

[45] K. F. H. Group, Transit Capacity and Quality of Service Manual, Transport Research Board of the National Academy Press, Washington, DC, 2013, (TCRP Report 100). 


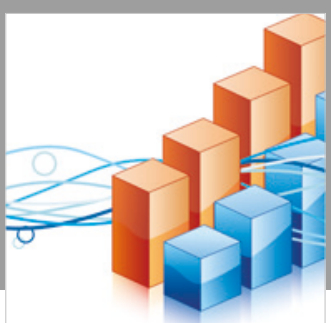

Advances in

Operations Research

\section{-n-m}
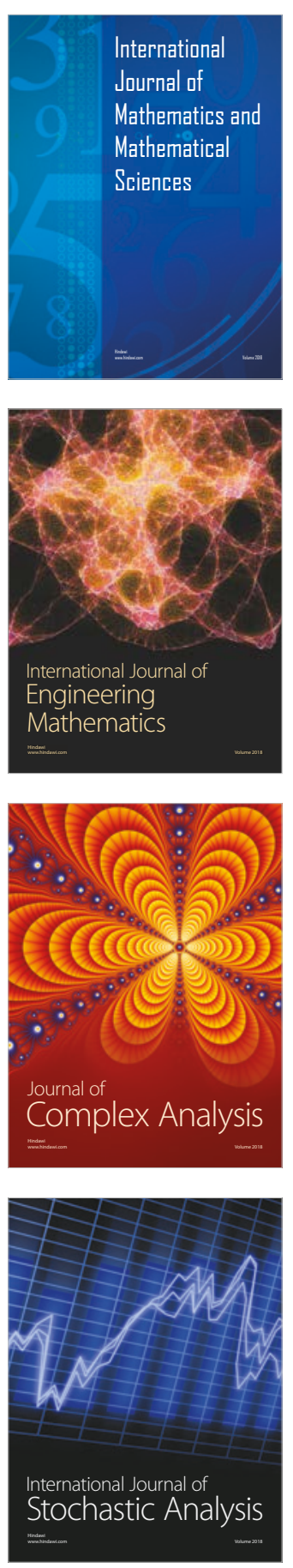
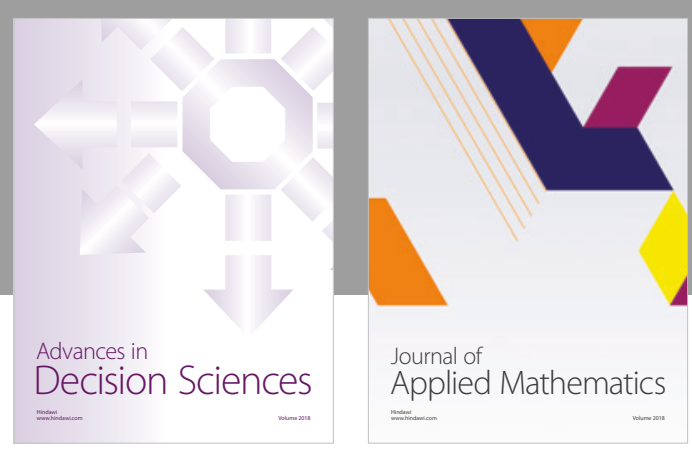

Journal of

Applied Mathematics
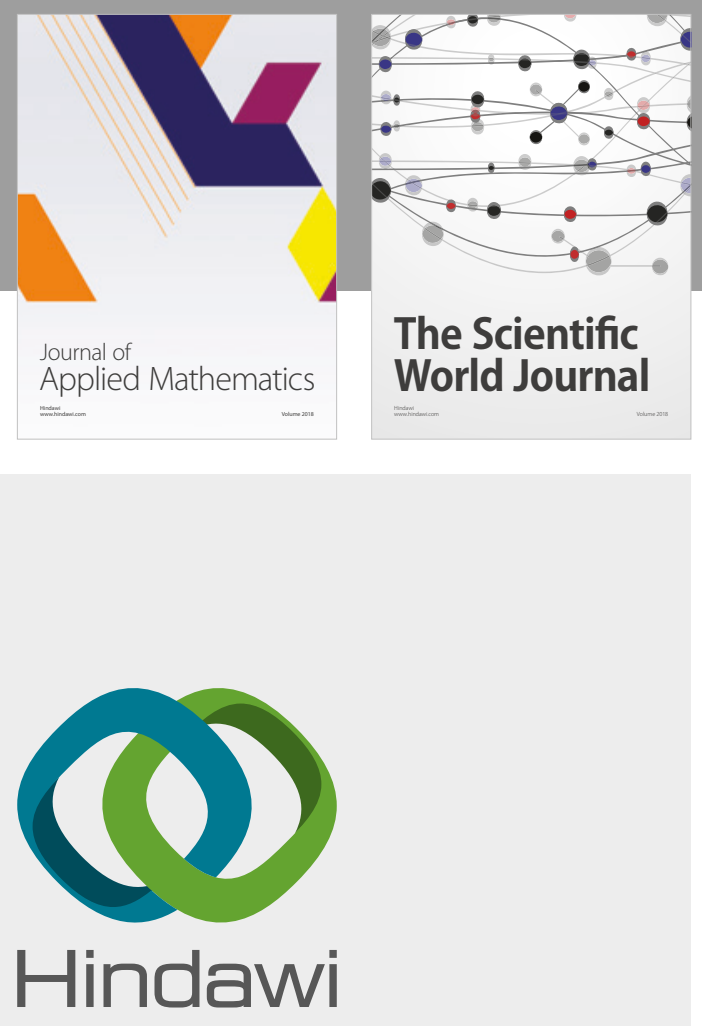

Submit your manuscripts at

www.hindawi.com

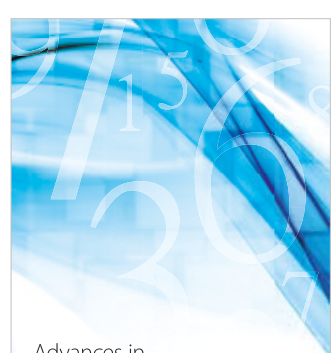

Advances in
Numerical Analysis
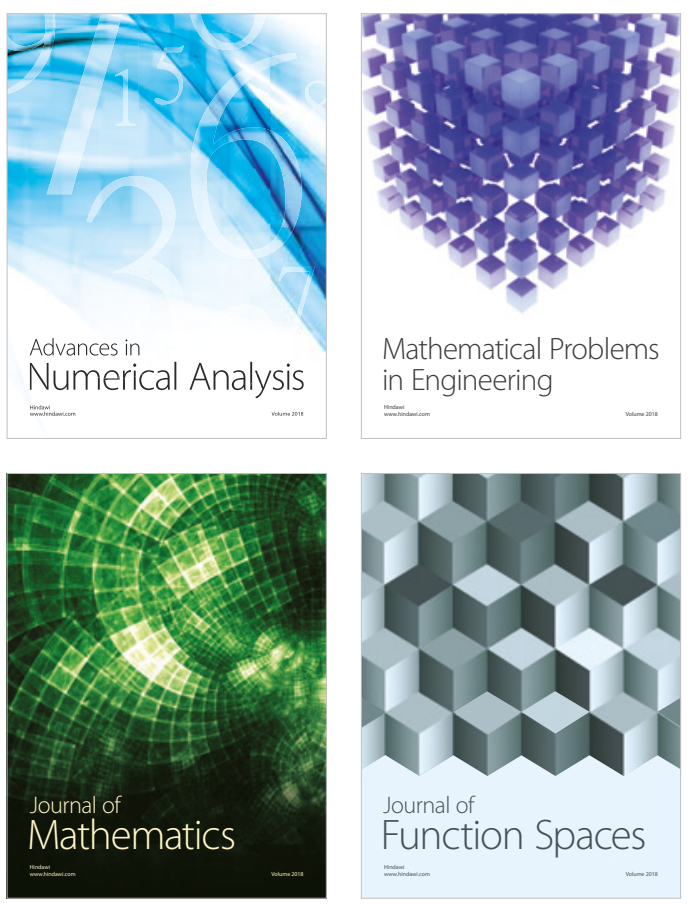

Mathematical Problems in Engineering

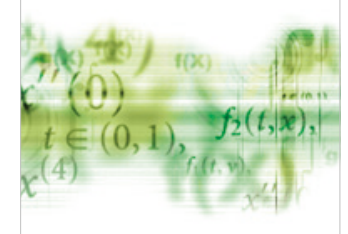

International Journal of

Differential Equations

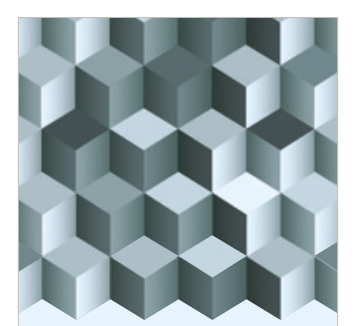

Journal of

Function Spaces
The Scientific

World Journal

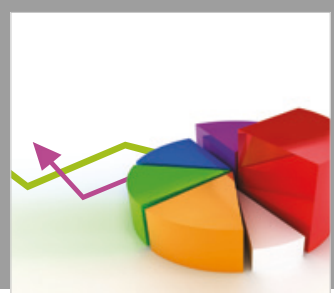

Journal of

Probability and Statistics
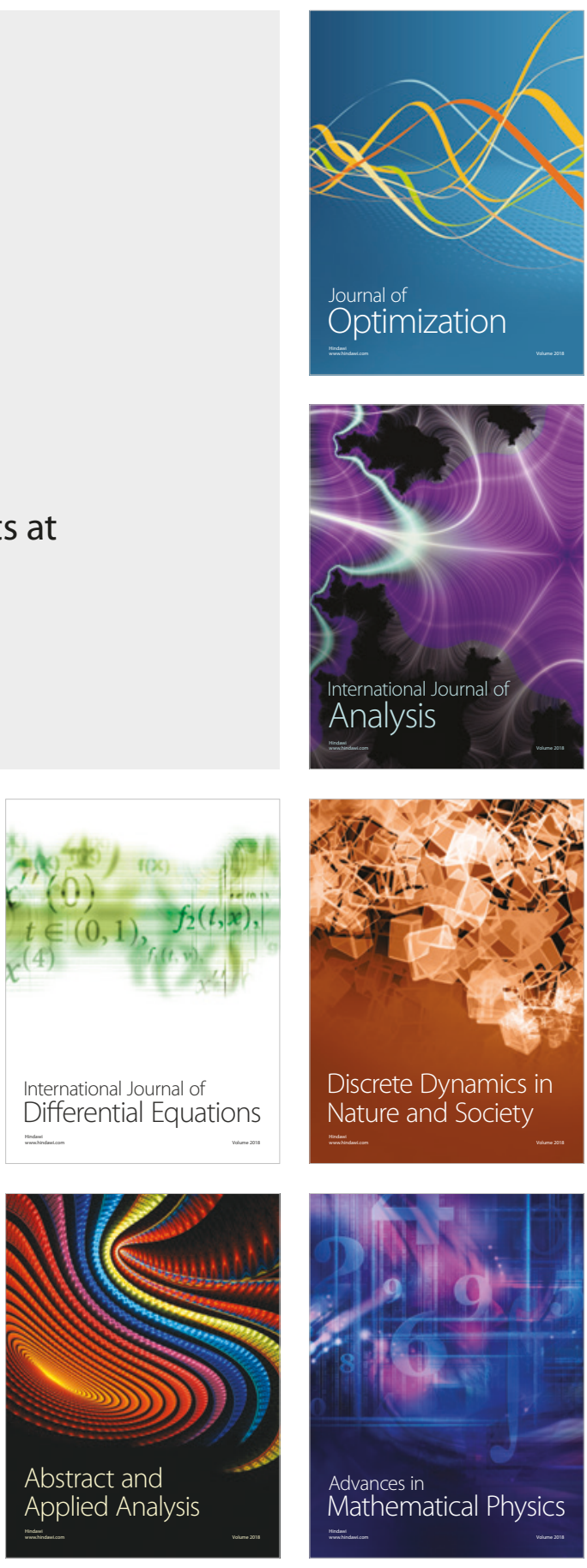